\title{
PLIN2 Mediates Neuroinflammation and Oxidative/ Nitrosative Stress via Downregulating Phosphatidylethanolamine in the Rostral Ventrolateral Medulla of Stressed Hypertensive Rats
}

\author{
Shutian Zhang ${ }^{1} * *$ \\ Li $\mathrm{Hu}^{2}$,* \\ Chengzhi Han (iD) \\ Renhui Huang' \\ Kokwin Ooi \\ Xinyi Qian' \\ Xiaorong Ren' \\ Dechang $\mathrm{Chu}^{3}$ \\ Haili Zhang (iD ${ }^{3}$ \\ Dongshu $\mathrm{Du}^{4}$ \\ Chunmei Xia (iD) \\ 'Department of Physiology and Pathophysiology, \\ School of Basic Medical Sciences, Fudan University, \\ Shanghai, 200032, People's Republic of China; \\ ${ }^{2}$ Department of Cardiology, Renji Hospital, School \\ of Medicine, Shanghai Jiaotong University, Shanghai, \\ 200240, People's Republic of China; ${ }^{3}$ College of \\ Agriculture and Bioengineering, Heze University, \\ Heze, 274000, People's Republic of China; ${ }^{4}$ School \\ of Life Science, Shanghai University, Shanghai, \\ 200444, People's Republic of China \\ *These authors contributed equally to this work
}

Correspondence: Chunmei Xia Department of Physiology and Pathophysiology, School of Basic Medical Sciences, Fudan University, Shanghai, 200032, People's Republic of China

Tel +86 21 542376 I2-805

Email cmxia@fudan.edu.cn

Dongshu Du

School of Life Science, Shanghai

University, Shanghai, 200444, People's

Republic of China

Emailsdhzdds@I63.com
Purpose: Oxidative/nitrosative stress, neuroinflammation and their intimate interactions mediate sympathetic overactivation in hypertension. An immoderate inflammatory response is characterized not only by elevated proinflammatory cytokines (PICs) but by increases in mitochondrial dysfunction, reactive oxygen species (ROS), and nitric oxide (NO). Recent data pinpoint that both the phospholipid and lipid droplets (LDs) are potent modulators of microglia physiology.

Methods: Stress rats underwent compound stressors for 15 days with PLIN2-siRNA or scrambled-siRNA (SC-siRNA) administrated into the rostral ventrolateral medulla (RVLM). Lipids were analyzed by mass spectroscopy-based quantitative lipidomics. The phenotypes and proliferation of microglia, LDs, in the RVLM of rats were detected; blood pressure (BP) and myocardial injury in rats were evaluated. The anti-oxidative/nitrosative stress effect of phosphatidylethanolamine (PE) was explored in cultured primary microglia.

Results: Lipidomics analysis showed that 75 individual lipids in RVLM were significantly dysregulated by stress [PE was the most one], demonstrating that lipid composition changed with stress. In vitro, prorenin stress induced the accumulation of LDs, increased PICs, which could be blocked by siRNA-PLIN2 in microglia. PLIN2 knockdown upregulated the PE synthesis in microglia. Anti-oxidative/nitrosative stress effect of PE delivery was confirmed by the decrease of ROS and decrease in 3-NT and MDA in prorenin-treated microglia. PLIN2 knockdown in the RVLM blocked the number of $\mathrm{iNOS}^{+}$and $\mathrm{PCNA}^{+}$microglia, decreased BP, alleviated cardiac fibrosis and hypertrophy in stressed rats.

Conclusion: PLIN2 mediates microglial polarization/proliferation via downregulating PE in the RVLM of stressed rats. Delivery of PE is a promising strategy for combating neuroinflammation and oxidative/nitrosative stress in stress-induced hypertension.

Keywords: lipid droplets, PLIN2, phosphatidylethanolamine, lipidomics, microglial expansion, neuroinflammation, stress, RVLM, oxidative/nitrosative stress

\section{Introduction}

Evidence shows that chronic stress is one of the important pathogenic factors of hypertension and target organ damage. ${ }^{1,2}$ Stress leads to activation of the HPA axis and increased sympathetic drive of cardiovascular center, which eventually increases blood pressure. Activation of microglia is one of the most significant cerebral changes induced by chronic stress. ${ }^{3,4}$ There is accumulating evidence that stress induce gliosis and inflammation in the rodent RVLM. ${ }^{5-7}$ It is noted that an 
excessive inflammatory response is featured not only by elevated inflammatory cytokines, but also by increases in mitochondrial dysfunction, reactive oxygen species (ROS), and nitric oxide (NO). Overactive brain local renin-angiotensin system (RAS), oxidative/nitrosative stress, and neuroinflammation in concert play a pivotal role in triggering augmenting sympathetic activity in the centers. $^{8-12}$ We previously reported that stress induced mitochondrial damage in the microglia of RVLM. ${ }^{13,14}$ Microglial mitophagy-lysosome dysfunction mediates the activation of intracellular NLRP3 inflammasome in stress hypertension mice, thus promoting the release of proinflammatory factors. ${ }^{13,14}$ In vitro, we found that stressed microglia showed increased mitochondria ROS (mitoROS). ${ }^{15}$ However, the molecular mechanisms of microglia M1 polarization and oxidative/nitrosative stress induced by stress and its effects on the hypertensive cardiac injury are not fully understood.

A variety of blood-derived and peripheral-derived stimuli activate microglia, which mediate the formation of cardiac sympathetic center inflammation. ${ }^{16,17}$ In addition to M1 pro-inflammatory activation and M2 antiinflammatory activation, which are widely concerned, neuroinflammation is also accompanied by microglia proliferation, resulting in the formation of microgliosis. ${ }^{18,19}$ It was confirmed that chronic stress and various metabolic stress can induce the proliferation and activation of microglia in the cardiac sympathetic center such as paraventricular nucleus (PVN). ${ }^{20-23}$

Recent data pinpoint that both the lipid droplets and phospholipid are potent modulators of microglia physiology. ${ }^{24-26}$ On the one hand, phospholipid provides a metabolic basis for the proliferation and activation of stress-related inflammatory cells, and on the other hand, it also acts as a pro-inflammatory mediator to mediate neuroinflammation through oxidative stress or Toll-like receptors (TLRs)-dependent pathways. ${ }^{27-31}$ Phosphatidylethanolamine (PE) and phosphatidylcholine (PC) are the two most abundant phospholipids in eukaryotic cells. Changes in the contents of PE and PC are sensitively regulated by chronic stress and exercise, and the abnormality of their conversion/ratio is also involved in the pathogenesis of metabolic syndrome. ${ }^{32-35}$ It has been found that the decrease of PE or the increase of PE to PC conversion is involved in the incidence of non-alcoholic fatty liver disease and obesity. ${ }^{36-38}$ It was reported that chronic stress resulted in decreased PE synthesis in the brain and in vitro administration of PE protected neurotoxicity due to stress hormone corticosterone. $^{32,39}$ Dysregulation of phospholipid synthesis and transformation is also involved in the inflammatory activation of macrophages and the release of pro-inflammatory factors such as IL-1 $\beta$, which suggests that the dysregulation of phospholipid metabolism represented by PE downregulation may be involved in the occurrence of neuroinflammation caused by stress. ${ }^{40,41}$ The potential anti-inflammatory and antioxidant effects of PE delivery need to be further verified.

Lipid droplets (LDs) are the most important organelles in regulating lipid metabolism. On the one hand, lipid droplets act as storage organelles for neutral fatty acids and provide substrates for phospholipid synthesis. The membrane of lipid droplets, on the other hand, acts as the functional docking site for the enzymes involved in phospholipid synthesis. ${ }^{42}$ While the perilipins, the lipid droplet membrane proteins, perform the function of recruitment of the enzymes involved in phospholipid synthesis. Highly proliferating cells, including cancer cells and inflammatory cells, constantly contain a large number of LDs, which provide a substantial basis for cell proliferation and the production of inflammatory signaling molecules. ${ }^{43-45}$ Inhibition of LDs accumulation regulates the metabolic pattern of proliferating cells and partially block cell proliferation signals. ${ }^{46-48}$ Perilipins are the important structural protein of LDs membrane, which separates the hydrophobic lipid core from the cytoplasm and participates in the maintenance of LDs homeostasis. PLIN2 is an inherent perilipin protein, and its expression and phosphorylation are involved in lipolysis, lipophagy, lipid droplet fusion, and signal transduction. ${ }^{49-52}$ PLIN2 activation-mediated LDs accumulation was observed in microglia activated by various inflammatory stimuli, which was closely related to the production of ROS and inflammatory cytokines. ${ }^{24,53,54}$ Our preliminary data showed that there was LDs accumulation in the stressed microglia, furthermore, the lipidomics analysis showed that PE was the most downregulated lipid in the RVLM of stressed rats, demonstrating that lipid composition changed with stress.

We previously reported that prorenin, a component of the RAS system, and HMGB1, a stress response molecule, mediated mitochondrial damage and autophagy blockade in RVLM microglia of SIH. ${ }^{13,14}$ The findings of mitochondrial damage and mitophagy drive us to further explore the molecular mechanism of stress-induced microglial activation from the perspective of metabolic remodeling. In our present study, we confirmed that PE deficiency induced by PLIN2 was involved in microglia polarization/proliferation and oxidative/nitrosative stress in RVLM. Exogenous PE supplementation effectively blocked 
prorenin-induced oxidative/nitrosative stress in cultured microglia. The centrally PLIN2 siRNA administration blocks the neuroinflammation, and then reverses the elevated blood pressure and myocardial injury caused by stress.

\section{Materials and Methods}

\section{Reagents and Antibodies}

Nitrotyrosine ELISA kit (ab113848) and Phosphatidylethanolamine Assay Kit (ab241005) was purchased from Abcam (Cambridge, MA). L- $\alpha$ phosphatidylethanolamine (PE) (L130310) was purchased from Aladdin (Shanghai, China). Mito-SOX (M36008), BODIPY probe (D3835) and WGA (W11261) were purchased from Thermo Fisher Scientific (Waltham, USA). GSH and GSSG Assay Kit (S0053), Lipid Peroxidation MDA Assay Kit (S0131M), Hematoxylin and Eosin Staining Kit (C0105M) were purchased from Beyotime (Shanghai, China). Masson's trichrome staining kit (DC0032) was purchased from Leagene (Beijing, China). NE ELISA kit (E-EL-0047c) was purchased from Elabscience (Wuhan, China). SC-siRNA (sc-37007); PLIN2siRNA (sc-270227) were purchased from Santa Cruz Biotechnology (California, USA). Antibodies: PLIN2 (ab108323), iNOS (ab178945), Iba-1 (ab153696), Iba-1 (ab283342), PGP 9.5 (ab72911), TNF- $\alpha$ (ab205587), PCNA (ab29), 3-Nitrotyrosine (ab61392) and IL-1 $\beta$ (ab254360) were from Abcam (Cambridge, MA). c-fos (\#2250, CST) was purchased from Cell Signaling Technology (Beverly, MA). 4-Hydroxynonenal (MA5-27570) was purchased from Thermo Fisher Scientific (Waltham, USA).

\section{Animals and Preparation}

Male adult Sprague-Dawley rats (8 weeks old, 250-300 g) were obtained from the Animal Laboratory Center of Fudan University. All experimental procedures conformed to the guidelines of the Institutional Ethics Committee of Fudan University and approved by Fudan University Animal Care Committee. All efforts were made to minimize the number of rats used and reduce their suffering. The rats were housed under a 12-h light/dark cycle in a temperature-controlled animal lab at $24^{\circ} \mathrm{C}$ with standard food and tap water ad libitum. The experimental design is shown in Figure 1. SCsiRNA or PLIN2-siRNA was microinjected into the RVLM of rats for centrally interfering the genes.

RVLM stereotaxic surgery and microinjection were performed as described in our previous study. ${ }^{5}$ Briefly, the rats were anesthetized intraperitoneally at a dose of $7 \mathrm{~mL} / \mathrm{kg}$ with a mixture anesthetic that was comprised of $140 \mathrm{~g}$ urethane, $7 \mathrm{~g}$ chloralose, and $7 \mathrm{~g}$ borax dissolved in $1 \mathrm{~L}$ normal saline. The head of rats were placed on a stereotaxic apparatus (Neurostar Tubingen, Germany) and flexed to an angle of $45^{\circ}$. The animals were intubated with polyethylene tubes and spontaneously breathed room air. The occipital bone was removed and the fourth ventricle was exposed of the rat. A glass micropipette was made and inserted into the RVLM (1.5-1.9 mm ahead of the obex, 1.5-2.0 $\mathrm{mm}$ right of the midline, and 6.6$7.0 \mathrm{~mm}$ deep from the dorsal surface of the cerebellum) as prescribed in the atlas of Paxinos and Watson. ${ }^{55}$ For knock down of PLIN2 in RVLM, rats were anesthetized and received bilateral RVLM microinjections of PLIN2 siRNA $(0.3 \mu \mathrm{g}$ in $0.7 \mu \mathrm{L} 10 \mathrm{mM}$ JetSITM). A scrambled siRNA was used as the control. After the injection was complete, the skull was closed with bone wax, and the surface of the skull was disinfected. Then the skin was interrupted sutured, and the incision was disinfected. The rats' body temperature was maintained at $37^{\circ} \mathrm{C}$ with heating pads until they were awake from anesthesia. Procaine penicillin was injected (1000 IU, i.m.) into the rats postoperatively.

Compound stresses were given to the rats as described in our previous publications. Briefly, rats were set in a $22 \mathrm{~cm} \times$ $22 \mathrm{~cm} \times 28 \mathrm{~cm}$-sized cage and underwent intermittent electric shocks $(35-75 \mathrm{~V}, 0.5 \mathrm{~ms}$ in duration) every 2-30 $\mathrm{s}$ controlled by a computer. Synchronously, the noise (range, 88-98 dB) produced by a buzzer was given to the rats as the conditioned stimulus. ${ }^{6}$ The rats were subjected to stress for $2 \mathrm{~h}$ twice/day for 15 consecutive days. The sham control rats underwent sham stress; by which they were put into the same size cage for the same period of time, but they were not subjected to foot-shocks or noise stress.

\section{Lipidomics}

The RVLM from 8 stressed rats and 8 sham rats were used for lipidome analyses. The lipidomics detection and analyses were performed by OE biotech (Shanghai, China) as described in detail in the work by $\mathrm{Wu}$ et al and Chen et al. ${ }^{56,57}$ To explain briefly, dichloromethane/methanol $(2 / 1, v / v)$ solution was used to extract lipids after homogenization with pure water. Lysophosphatidylcholine $(17: 0,0.01 \mathrm{mg} / \mathrm{mL})$ and L-2-chlorophenylalanine $(0.3 \mathrm{mg} /$ $\mathrm{mL}$ ) were used as the internal standards. Isopropanol/ methanol (1/1, v/v) were used as the sol-vents. Lipidomic analyses were conducted on an Ultimate-3000 


\begin{tabular}{l|l}
$\begin{array}{l}\text { Experiment } 1 \\
\text { Sham or Stress }\end{array}$ & Quantitative lipidomics(n=8/group) \\
\hline 2 weeks & $\begin{array}{l}\text { Transmission electron microscopy ( } \mathrm{n}=6 / \text { group }) \\
\text { Immunofluorescent staining (n=6/group) }\end{array}$
\end{tabular}

\begin{tabular}{l|l|l}
$\begin{array}{l}\text { Experiment } 2 \\
\text { Sham or Stress }\end{array}$ & $\begin{array}{l}\text { Microinjections } \\
\text { siRNA; (n=6/group) }\end{array}$ & $\begin{array}{l}\text { Western blot; Immunofluorescent staining } \\
\text { (n=3-5/group); HR, BP, NE measurement; } \\
\text { cardiac function evaluation (n=6/group) }\end{array}$ \\
\hline 2 weeks & 1 week & 1 day
\end{tabular}

\begin{tabular}{l|l}
$\begin{array}{l}\text { Experiment } 3 \text { in vitro (primary microglia) } \\
\begin{array}{l}\text { Vehicle or Prorenin or Prorenin } \\
\text { +phosphatidylethanolamine or } \\
\text { Prorenin+PLIN2-siRNA }\end{array}\end{array}$ & $\begin{array}{l}\text { Western bolt; Immunofluorescent } \\
\text { BODIPY staining; Mito-SOX/nitrosative } \\
\text { stress measurement (n=3-5/group) }\end{array}$ \\
\hline \multicolumn{1}{c}{6 hours } & 1 hour
\end{tabular}

Figure I The experimental flow chart of the study.

UPLC system coupled to a Q-Exactive hybrid quadrupoleOrbitrap MS system (Thermo Scientific), where a $100 \times 2.1-\mathrm{mm}$ hypersil GOLD $1.9-\mu \mathrm{m}$ C18 column (Thermo Scientific) was used. All raw data were acquired using the software Xcalibur (version 3.0, Thermo Scientific). LipidSearch (Version 4.0, Thermo Scientific) was employed for lipid identification and quantification.

\section{Microglia Isolations and ex vivo Treatments}

Primary microglia were cultured as in our previous publication. Briefly, the medulla oblongata containing the RVLM was taken from 1-2-day-old Sprague-Dawley rats following sacrifice via decapitation. ${ }^{13}$ The site of RVLM was identified in accordance with the atlas of Watson and Paxinos. ${ }^{55}$ Both sides of the RVLM (as above-mentioned) were collected using micropunches and then the minced tissue was incubated in the dissecting medium containing glucose, bovine serum albumin (BSA) and HEPES, as well as $0.025 \%$ trypsin at $37^{\circ} \mathrm{C}$ for $20 \mathrm{~min}$. Then, cells were plated at $3 \times 105$ cells $/ \mathrm{cm} 2$ in Dulbecco's modified Eagle's medium (DMEM) with GlutaMax and high glucose (4.5 g/ L), supplemented with $10 \%$ fetal bovine serum, $0.1 \mathrm{mg}$ / $\mathrm{mL}$ streptomycin and $100 \mathrm{U} / \mathrm{mL}$ penicillin in poly1-lysine-coated $75 \mathrm{~cm} 2$ culture flasks. On day 9, the cells were resuspended after centrifugation $(150 \times \mathrm{g}$ for 10 min). Cell viability was evaluated by trypan blue exclusion. The purity of cultured microglia was $>95 \%$ when identified by specific microglia marker staining. The in vitro experiment design is shown in Figure 1. The siRNA is purchased from Santa Cruz Biotechnology and was transfected into microglia to knock down PLIN2.

\section{Hemodynamic Measurements (Blood Pressure, HRV, Echocardiogram)}

The rats were anesthetized with sodium pentobarbitone $(50 \mathrm{mg} / \mathrm{kg}$ ) intraperitoneally (i.p.). The cardiac diastolic and systolic functions of the rats were evaluated by transthoracic echocardiography before the rats were sacrificed. The images were processed at $21 \mathrm{MHz}$ using a MS-250 transducer operated by a Vevo 2100 Color Doppler Ultrasound Scanner (FUJIFILM VisualSonics Inc., Toronto, ON, Canada). BP was measured via a femoral artery cannula using a pressure transducer and a polygraph (Powerlab, AD instrument, Australia) for 5 minutes. The limb lead was made to record the ECG. The analysis of heart rate variability (HRV) is also derived from the analysis module in POWER LAB.

\section{Histological Evaluation of Cardiac Tissue}

Masson's trichrome staining, hematoxylin-eosin (HE) staining and Alexa Fluor 488 conjugate of Wheat germ agglutinin staining (WGA, cell membrane staining) was used to in $5-\mu \mathrm{m}$ thin myocardial sections. Interstitial 
fibrosis (excluding vascular spaces) was evaluated on Masson's trichrome stained sections. Briefly, the area of fibrotic tissue to total tissue area of the myocardium was determined by ImageJ software (NIH, Bethesda, MD, USA) using thresholding on red-green-blue stack images of Masson's trichrome sections by two independent observers. HE and/or WGA stained sections were used to evaluate cardiomyocyte hypertrophy by measuring the crosssection area as described previously. ${ }^{58}$

\section{Immunofluorescent Staining}

Immunofluorescence staining was performed as we previously described. Following the rat's deep anesthetization, it was perfused by $300 \mathrm{~mL}$ of $0.01 \mathrm{M}$ PBS (pH 7.4) via left ventricle and was subsequently perfused by $300 \mathrm{~mL}$ of $4 \%$ paraformaldehyde solution dissolved in 0.1M PBS. The RVLM sections were post-fixated for $4 \mathrm{~h}$, and then they were placed in the gradient of $20 \%$ and $30 \%$ sucrose at $4^{\circ} \mathrm{C}$ for overnight dehydration, respectively. The $30-\mu \mathrm{m}$ thick free-floating coronal sections containing the RVLM were subsequently washed in PBS and then permeabilized with $0.3 \%$ Triton $\mathrm{X}-100$ for $30 \mathrm{~min}$ followed by incubation with $5 \%$ goat serum for $1 \mathrm{~h}$ at $37^{\circ} \mathrm{C}$ to block non-specific protein. The sections were incubated with primary antibody [antibodies: PLIN2, iNOS, TNF- $\alpha, \quad$ IL-1 $\beta$, PCNA, 3-Nitrotyrosine, 4-Hydroxynonenal, c-fos, Iba-1 (a microglia marker), and/or PGP 9.5 (a neuronal marker)] for overnight at $4{ }^{\circ} \mathrm{C}$ then with the secondary antibody [The Donkey Anti-Goat IgG H\&L (Alexa Fluor 488), Donkey Anti-Goat IgG H\&L (Cy5), Donkey Anti-Rabbit IgG H\&L (Alexa Fluor 647), and Donkey Anti-Rabbit IgG H\&L (Alexa Fluor 488)] for $2 \mathrm{~h}$ at room temperature, respectively. The sections were observed under FluoView FV300 laser scanning confocal microscope (Zeiss, Carl Zeiss, Germany). The immunofluorescent images were processed using ImageJ software.

\section{Lipid Droplets Analysis}

$\mathrm{BODIPY}^{+}$staining was used to evaluate the LDs from six randomly selected visual fields (maximum projection of the $\mathrm{z}$-stack across the whole section). The number of LDs were manually counted in RVLM by two independent observers. To measure the average size of lipid droplets, BODIPY $^{+}$signal was analyzed using the "Analyze Particles" function of ImageJ 1.45 s (ImageJ website: http://imagej.nih.gov.laneproxy.stanford.edu/ij/) for six randomly selected visual fields.

\section{Western Blot}

Total proteins were extracted from the RVLM tissue following immunoblotting procedures as we previously described. ${ }^{13-15}$ The RVLM tissue was homogenized in lysis buffer with $1 \%$ NP40 and $1 \mathrm{mmol} / \mathrm{L}$ PMSF. Protein samples of the same amount from each rat were extracted from RVLM homogenates to analyze protein expression by Western blot. In brief, the protein samples (20 $\mu$ g each) were subjected to SDS/PAGE in $8-12 \%$ gradient gel (Invitrogen, Carlsbad, CA) and transferred to PVDF membrane. Primary antibodies were incubated and followed by incubation with horseradish peroxidaseconjugated goat anti-rabbit IgG or goat anti-mouse IgG. The amount of protein was assessed by ECL detection reagents (WBKLS0050; Millipore) and the immunostaining band was visualized and quantitated by an automatic chemiluminescence image analysis system (Tanon-5200; Tanon Science \& Technology, Shanghai, China). The data were normalized by developing the $\beta$-actin as loading control. The concentration of all the antibodies was 1:1000 except for $\beta$-actin (1:5000).

\section{Transmission Electron Microscopy}

The procedures were reported as in our previous publication. ${ }^{14}$ In brief, RVLM tissue was fixed, osmicated, rinsed with phosphate buffer, dehydrated, and embedded in epoxy resin, which was allowed to polymerize for $24 \mathrm{~h}$ at $70^{\circ} \mathrm{C}$. Blocks containing RVLM were sectioned using an ultramicrotome (Ultracut; Leica) at 70-80 nm, which were collected on grids and stained with uranyl acetate and lead citrate. Grids were examined under a transmission electron microscope (H-700; Hitachi, Tokyo, Japan) at $80 \mathrm{kV}$.

\section{Mitochondrial ROS Measurement}

Mitochondrial superoxide anions were measured following the manufacturer's instructions of MitoSOX Red kit (Thermo Fisher Scientific) to reflect the mitochondrial ROS level. Briefly, $5 \mu \mathrm{M}$ MitoSOX reagent working solution was prepared and incubated cells for 10 minutes at $37^{\circ} \mathrm{C}$, protected from light. Cells without the MitoSOX reagent incubation were used to subtract the background. Mean fluorescence intensity was determined, and all samples were normalized to control ones.

\section{Phosphatidylethanolamine (PE) Concentration Analysis}

PE concentration in the RVLM of rats and/or microglia in vitro were performed using a PE assay kits (ab241005, Abcam) according to the manufacturer's instructions. 


\section{ELISA}

ELISA analysis was conducted according to the manufacturer's instructions. The plasmic NE concentration in the rats was measured by using NE ELISA kit (E-EL-0047c, Elabscience). 3-NT concentration in the supernatant of culture medium of microglia was measured using Nitrotyrosine ELISA kit (ab113848, Abcam).

\section{Statistical Analysis}

SPSS 11.0 software was applied to analyze the data obtained. Data were expressed as mean \pm standard error of the mean (SEM). The treatment group means were assessed by variance analysis followed by Tukey's multiple comparisons post hoc test (if variance was equal) or Dunnett T3 (if variance was not equal) using GraphPad Prism Software. P-values $<0.05$ were considered significant for all tests.

\section{Results}

\section{Stress Promotes Lipid Droplets} Accumulation in the RVLM of Rats

In an attempt to discover the ultrastructural differences between sham and stressed RVLM of rats, we analyzed their cytoplasmic content by transmission electron microscopy. We observed representative lipid droplets (LDs) were present in stressed but rarely in sham RVLM of rats. Furthermore, LDs were mostly adjacent to or contained in lysosomes (Figure 2A). To study whether dynamics of LDs in neuron/microglia of RVLM was altered in stressed rats, double immunofluorescent staining of BODIPY, a dye that specifically labels neutral lipids and is commonly used to detect LDs, with PGP9.5 (neuronal marker)/Iba-1 (microglial marker) was performed (Figure $2 \mathrm{~B}$ and $\mathrm{C}$ ). The results showed lot and larger LDs in the RVLM of stressed rats (Figure 2D and E). The percentage of BODIPY $^{+}$neuron/microglia in the RVLM was 3-fold higher in stressed compared with that of sham rats (Figure $2 \mathrm{~F}$ and $\mathrm{G}$ ). LDs were also immunoreactive with its constitute lipid droplet surface protein Perilipin 2 (PLIN2). We then detected PLIN2 expression in RVLM of rat brain sections (Figure $2 \mathrm{H}$ and I), we found that there was more counted PLIN2 ${ }^{+}$neuron/microglia in stressed than in that of sham rats (Figure 2J and K). Taken together, these data showed that stress induced the accumulation of LDs in the RVLM, accompanied by an increase in the expression of PLIN2.

\section{Stress Leads to PE Deficiency in the RVLM of Rats}

Lipid droplet is the regulatory organelle of cellular lipid metabolism. The accumulation of lipid droplet in microglia suggests that lipid metabolism disorder might participate in RVLM neuroinflammation. Untargeted lipidomic analysis was performed to investigate the impact of stress on the RVLM lipidome and its association with neuroinflammation. The unsupervised PCA showed an obvious separation between the sham and stress group with R2X [1] at 0.285 and R2X[2] at 0.194 (Figure 3A). A significant discrimination in metabolic phenotypes was further observed from the OPLS-DA mode with Q2 at 0.75 (Figure 3B). Further, PLS-DA mode verified the significant separation between the sham and stress group with R2 [Y] at 0.998 (Figure 3C). A total of 1341 individual lipid species were manually identified based on their MS/MS spectra and quantified (Figure 3D), among which, 75 individual lipids were significantly dysregulated by stress with the selection criterion of VIP $>1.0$ and $p$ value $<0.05$. Their relative expression level between sham and stress group were visualized in a heatmap based on cluster analysis (Figure 3E). Notably, the reduction in PE (20:0e/ 22:4) was the largest among the detected lipid. According to the KEGG pathway enrichment analysis (https://www.kegg.jp/), the identified lipid species were sorted into 17 metabolic pathways (Figure 3F). Among them, Glycerophospholipids metabolism showed the highest diversity, followed by choline and retrograde endocannabinoid metabolism.

\section{PLIN2 Knockdown Alleviated PE Deficiency in Stressed RVLM of Rats and/ or Prorenin-Treated Microglia}

To determine the effect of LDs on PE synthesis, we used siRNA to knockdown PLIN2 to reduce LDs accumulation in RVLM in vivo/microglia in vitro, and then quantified the PE concentration. The PE assay kit was used for the quantitation of $\mathrm{PE}$ in the RVLM/microglia. We found that intra-RVLM microinjections of PLIN2-siRNA robustly attenuated the protein levels of PLIN2 in the RVLM of stressed rats (Figure 4A and B), as compared with that of vehicle group. Immunofluorescent staining of PLIN2 was used to verify the knock down efficiency of PLIN2-siRNA in RVLM (Figure 4C and D). The result of PE assay kit test showed that stress induced PE deficiency in RVLM while PLIN2-siRNA inhibited its decline (Figure 4E). 
A

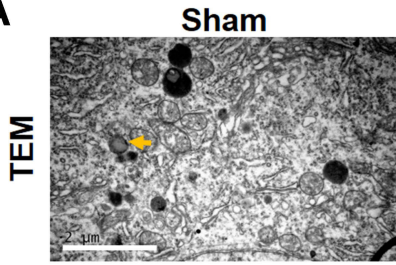

D

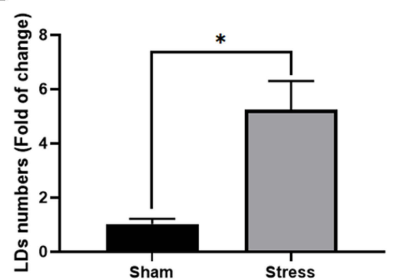

F
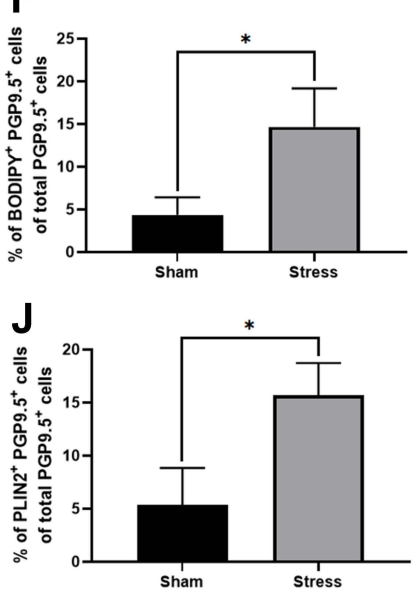

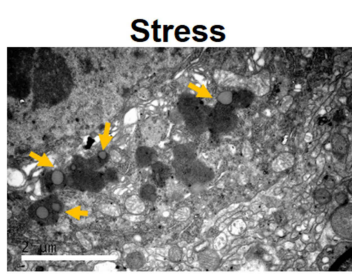

E

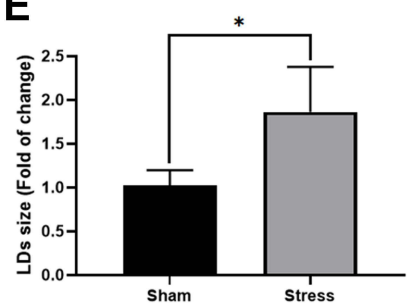

G

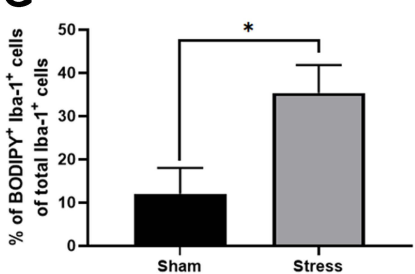

K

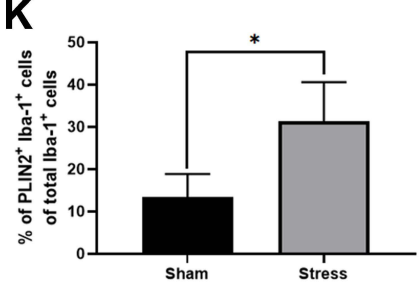

$\mathbf{B}_{\text {เ }}$
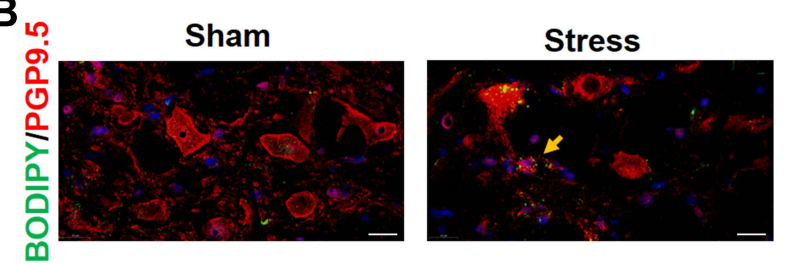

C
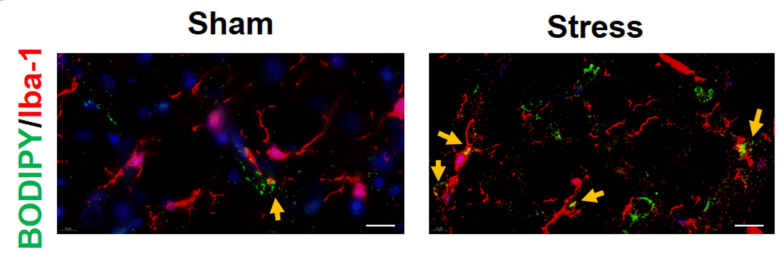

H
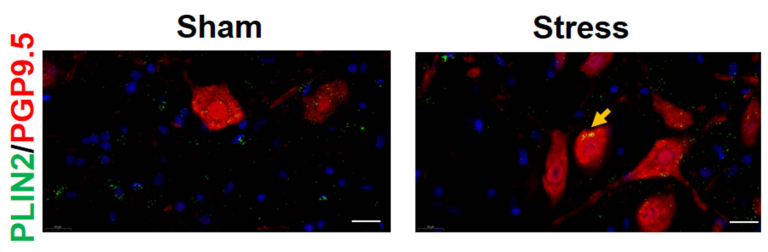

I
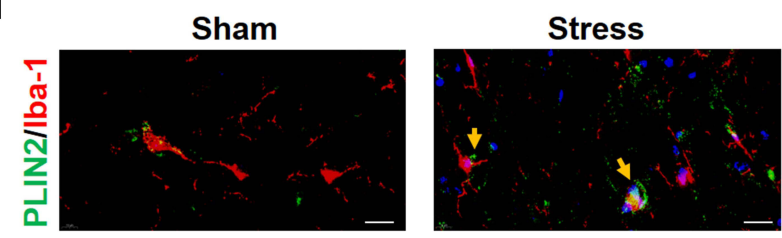

Figure 2 Stress promotes LDs accumulation in RVLM. (A) Representative transmission electron microscopic photomicrographs of RVLM in stress and sham rats. Yellow arrows indicate LDs. Scale bar $=2 \mu \mathrm{m}$. (B) Double immunofluorescent staining of BODIPY (a specific probe of LDs) with PGP9.5 (a neuronal marker) in the RVLM of rats. (C) Double immunofluorescent staining of BODIPY with Iba-I (a microglial marker) in the RVLM of rats. (D-G) Quantification of BODIPY lipid droplet numbers in the RVLM (D), average BODIPY ${ }^{+}$lipid droplet size in the RVLM (E), percent BODIPY ${ }^{+} /$PGP9. $5^{+}$cells $(\mathbf{F})$, and percent BODIPY ${ }^{+} /$lba- ${ }^{+}$cells $(\mathbf{G})$ in RVLM. (H) Double immunofluorescent staining of PLIN2 with PGP9.5 in the RVLM of rats. (I) Double immunofluorescent staining of PLIN2 with Iba-I in the RVLM of rats. (J and K) Percent PLIN2 ${ }^{+} /$PGP9. $5^{+}$cells $(\mathbf{J})$, and percent PLIN2 ${ }^{+} /$lba- $^{+}$cells $(\mathbf{K})$ in RVLM. Scale bar $=20 \mu \mathrm{m}$ in $(\mathbf{B}),(\mathbf{C}),(\mathbf{H})$ and $(\mathbf{I})$. Data are presented as the mean \pm SD. $n=6-8,{ }^{*} \mathrm{p}<0.05$, $t$-test.

Our previous studies have confirmed that prorenin is an important pro-inflammatory intermediate molecule in the activation of RVLM microglia induced by prohypertensive stress. Therefore, prorenin $(20 \mathrm{nmol} / \mathrm{L}$ for $24 \mathrm{~h}$ ) was used to stimulate microglia cells in all in vitro experiments of the present study. Transfection of cells with PLIN2-siRNA robustly diminished the PLIN2 expression (Figure 4F and G). Notably, after knock down PLIN2, LDs were reduced in number in microglia (Figure $4 \mathrm{H}$ and I), confirming that PLIN2 is a critical determinant of LDs accumulation in microglia. Further, with PE assay kit test, we found that prorenin drastically decrease $\mathrm{PE}$ in microglia while knockdown of PLIN2 suppressed these effects (Figure 4J). Altogether, these results suggest that the knockdown of PLIN2 attenuates PE deficiency in stressed RVLM/prorenin treated microglia.

\section{Delivery of PE Ameliorates}

\section{Prorenin-Induced Oxidative/Nitrosative Stress in Microglia}

Given the decreased PE in the stressed microglia, we speculated that $\mathrm{PE}$ play a potential role in microglial pathophysiology. Exogenous PE was delivered in prorenin-treated microglia to investigate whether PE deficiency was involved in microglial oxidative/nitrosative stress in vitro. We first used Mito-SOX probe to detect the level of ROS represented by mitochondrial 


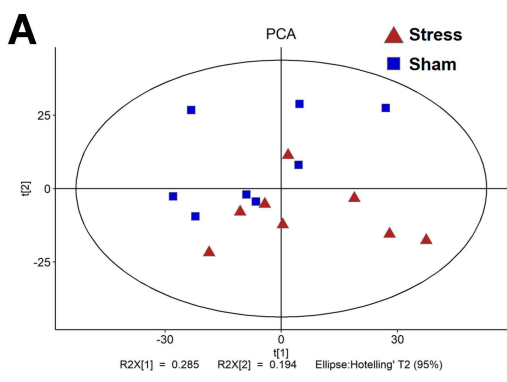

D

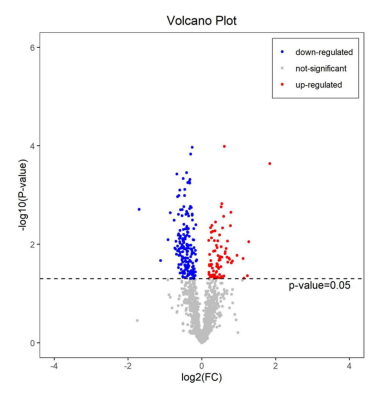

B

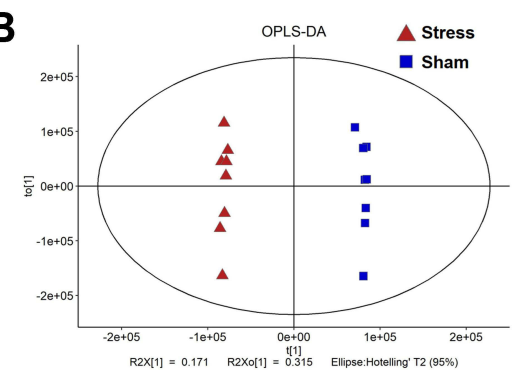

E

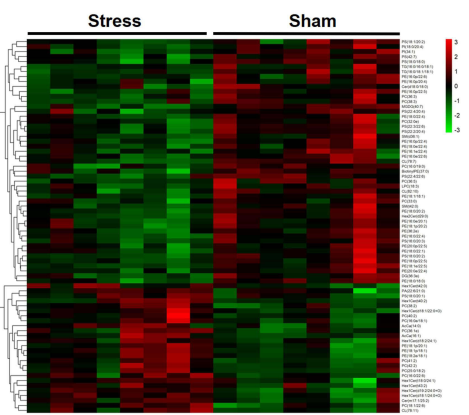

C

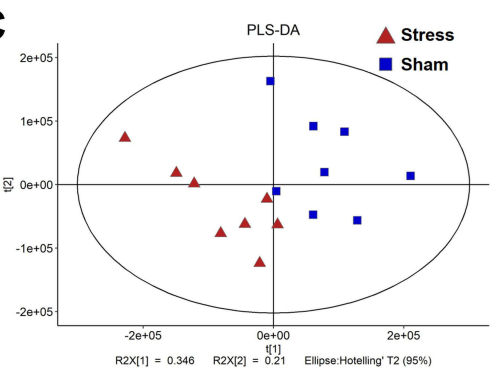

$\mathbf{F}$

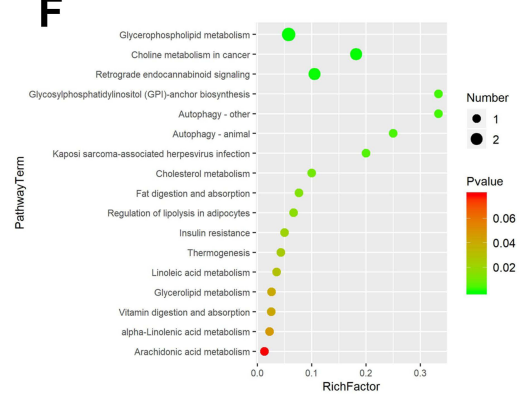

Figure 3 Stress leads to phosphatidylethanolamine deficiency in the RVLM. (A) PCA score plots, (B) OPLS-DA score plots and (C) PLS-DA score plots of sham and stress rats. (D) The volcano plot is represented by the log2 (fold change) of stress vs sham plotted against the -log 10 . (E) Heatmap of the 75 differential metabolites identified from the comparison of sham versus stress rats. The colors from green to red in the heatmap indicates metabolites elevation from low to the high. (F) Pathway enrichment analysis on the basis of the altered metabolites. Data are presented as the mean \pm SD. $n=8, p<0.05$, $t$-test.
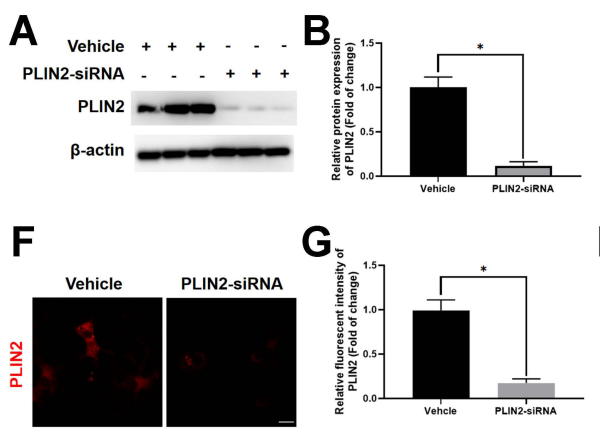
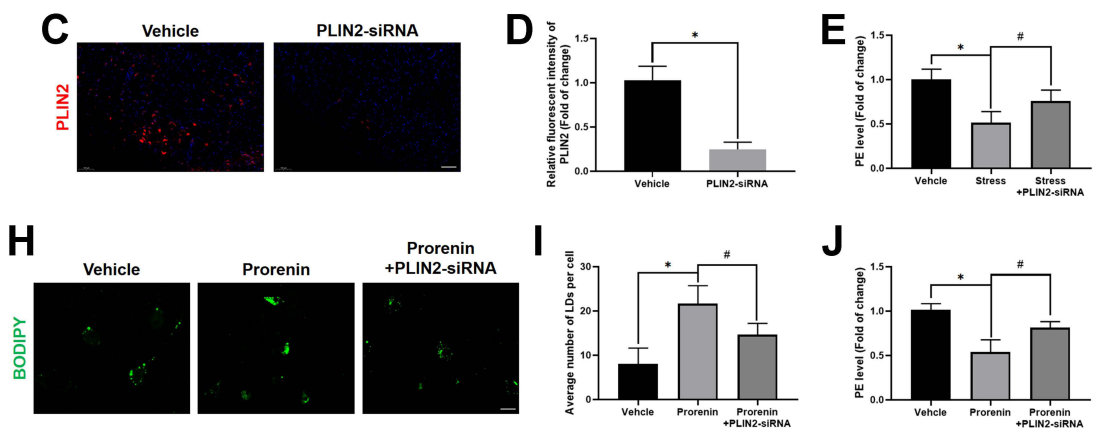

Figure 4 T PLIN2 knock down alleviated PE deficiency in stressed RVLM of rats and/or prorenin-treated microglia. (A and B) Western blot results showed that PLIN2 protein was deleted in the RVLM of PLIN2-siRNA microinjected rats. (C and D) Immuno-fluorescent staining also showed that PLIN2 protein in RVLM was deleted in PLIN2-siRNA microinjection rats. Scale bar $=100 \mu \mathrm{m}$. (E) The results of PE assay showed knock down PLIN2 alleviates stress-induced PE deficiency in RVLM of rats. (F and G) Immunofluorescent staining result showed PLIN2 deletion was achieved in the cultured microglia. Scale bar $=4 \mu \mathrm{m}$. (H and I) BODIPY staining showed PLIN2 knock down attenuated LDs accumulation in cultured microglia stimulated by prorenin. Scale bar $=4 \mu \mathrm{m}$. $(J)$ The results of PE assay further verified that knocked down of PLIN2 alleviated PE deficiency in the prorenin-treated microglia. Data are presented as the mean \pm SD. $n=6-8,{ }^{*} p<0.05$, ${ }^{\#} p<0.05, t$-test.

superoxide anions, and found that a large amount of ROS was produced in microglia by prorenin stimulation, while PE supplementation inhibited the ROS production (Figure $5 \mathrm{~A}$ and $\mathrm{B}$ ). The ratio of GSSG/GSH was measured to reflect the exhaustion of the antioxidant system. Our results showed that PE supplementation reduced the ratio of GSSG/GSH in prorenin-treated microglia (Figure 5C), indicating that PE supplementation increased the antioxidant capacity of microglia under stress. The level of nitrosative stress was evaluated using nitrotyrosine (3-nitrotyrosine, 3NT) competitive ELISA to determine the level of nitrotyrosine modified proteins. We observed that prorenin induced nitrosative stress in stressed microglia, which could be attenuated by PE supplementation (Figure 5D). Finally, we detected lipid peroxidation level with MDA kit, and found that supplementation of PE inhibited prorenininduced lipid peroxidation in microglia (Figure 5E). 
A
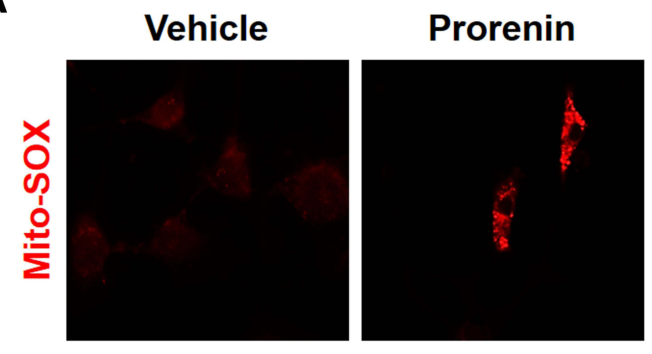

Prorenin + PE

C

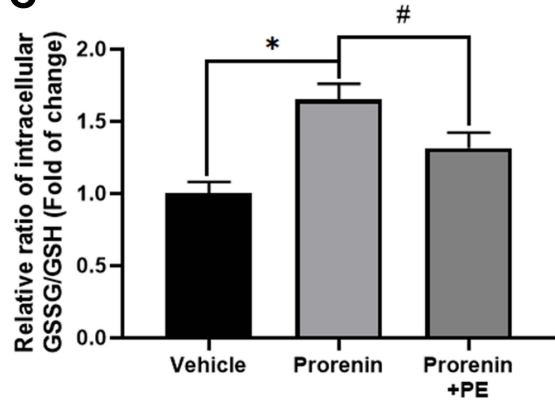

D

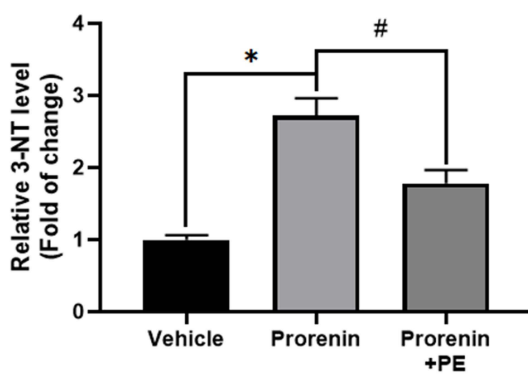

B :

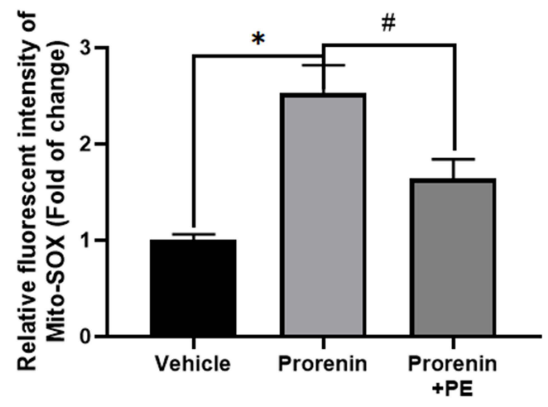

E

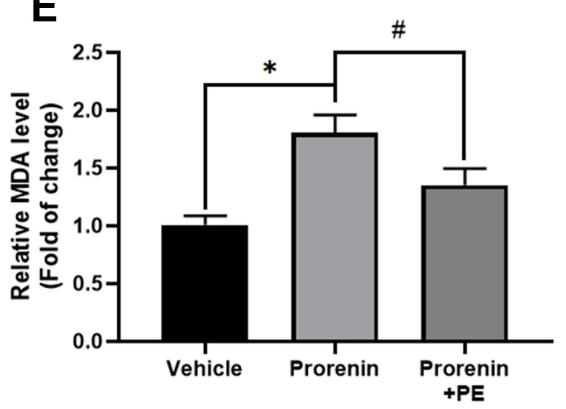

Figure 5 Delivery of exogenous PE ameliorates prorenin-induced oxidative/nitrosative stress in microglia. (A and B) Representative graph indicates fluorescent probe staining and intensity values quantification of mitoROS. Scale bar $=4 \mu \mathrm{m}$. (C) The GSSG/GSH ratio was detected using GSH and GSSG Assay Kit. The results indicate exogenous PE delivery attenuates prorenin-induced GSSG/GSH ratio increase in cultured microglia. (D) The level of nitrosative indicator 3-NT quantified using nitrotyrosine ELISA Kit. The results suggest delivery of PE ameliorates prorenin-induced nitrosative stress in cultured microglia. (E) The lipid peroxidation was measured using MDA kits. The data imply PE supplement alleviates lipid peroxidation in prorenin stimulated-microglia. Data are presented as the mean \pm SD. $n=3-5$, ${ }^{*}<0.05,{ }^{*}<0.05, t$-test.

\section{PLIN2 Knockdown Alleviates RVLM Oxidative/Nitrosative Stress in Stressed Rats in vivo}

4-Hydroxynonenal (4-HNE) is an $\alpha, \beta$ unsaturated hydroxyalkenal and an oxidative/nitrosative stress biomarker. Double immunofluorescence staining of 4-HNE and microglia marker Iba-1 showed that stress induced increased 4-HNE levels, which were inhibited by PLIN2-siRNA (Figure 6A and B). Besides, we also observed that PLIN2siRNA decreased the proportion of $4-\mathrm{HNE}^{+}$microglia (Figure 6C), suggesting that PLIN2-siRNA alleviated stress-induced oxidative stress in RVLM. Furthermore, immunofluorescent results showed that the nitrosative stress product 3-NT was increased in RVLM induced by stress, which was inhibited by PLIN2-siRNA (Figure 6D and E), and the proportion of $3-\mathrm{NT}^{+}$microglia in RVLM of rats under stress was decreased (Figure 6F). Furthermore, Western blot analysis showed an increase in Iba-1, 4-HNE and 3-NT expression in the RVLM of stressed rats compared with that of the sham group, while administration of PLIN2 siRNA significantly inhibited the increase of Iba-1, 4-HNE and 3-NT $(P<0.05$, Figure S1). These results indicated that knockdown of PLIN2 inhibited oxidative/ nitrosative stress of RVLM in rats.

\section{PLIN2 KnockDown Attenuates RVLM Microglial MI Polarization and Proliferation in Stressed Rats}

Next, we investigated whether microglial polarization and proliferation are regulated by PLIN2. We found that microinjection of PLIN2-siRNA decreases the proportion of $\mathrm{iNOS}^{+}$microglia (M1-polarization) in RVLM of stressed rats (Figure 7A and B). Consistently, PLIN2siRNA decreased the number of $\mathrm{PCNA}^{+}$(proliferation marker) microglia in RVLM of stressed rats (Figure 7C and D). Notably, proinflammatory cytokines, IL-1 $\beta$ and TNF- $\alpha$ expression was decreased by PLIN2-siRNA (Figure 7E-G). These data suggest that PLIN2 knockdown significantly reduced RVLM microglia inflammatory polarization and proliferation in stressed rats.

\section{PLIN2 Knockdown Alleviates}

\section{Sympathetic Overdrive and Lowers BP in}

\section{Stressed Rats}

Double immunofluorescent staining of neuronal activation marker c-fos with PGP9.5 in RVLM was performed to study pre-sympathetic neuron activation. The results showed that PLIN2-siRNA decreased $\mathrm{c}^{-} \mathrm{fos}^{+}$neuron 
A

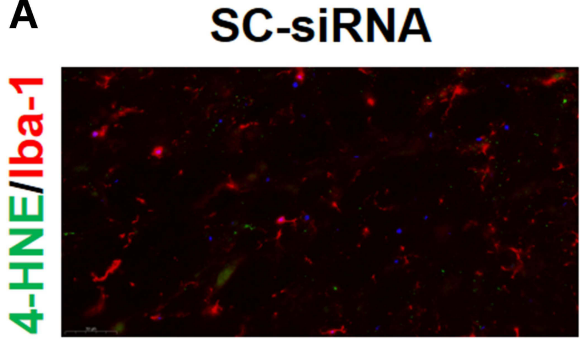

B

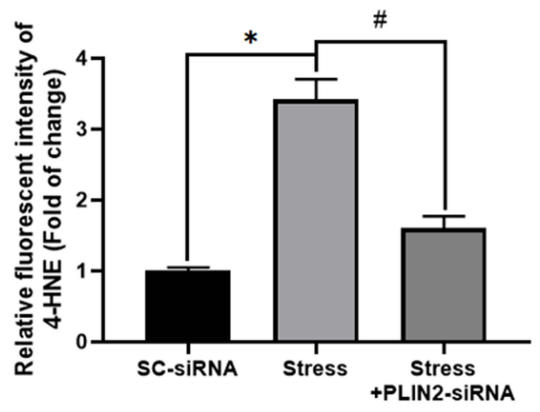

D

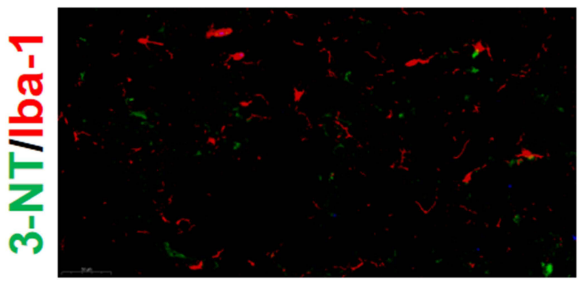

E

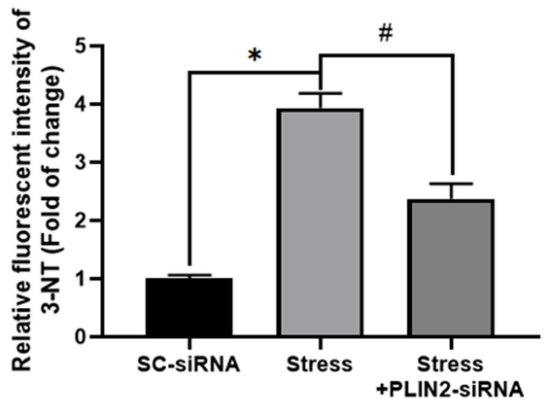

Stress

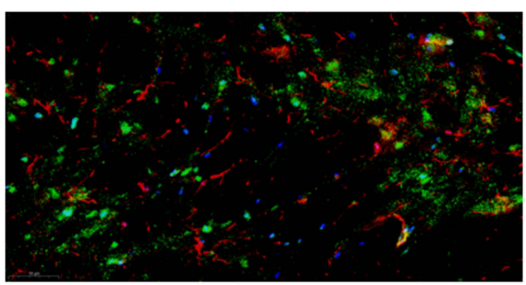

Stress + PLIN2-siRNA

C
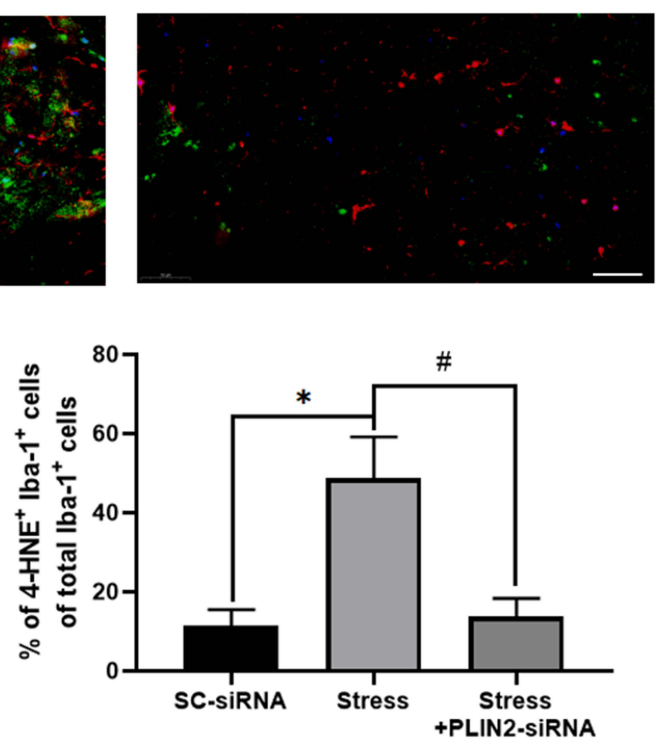
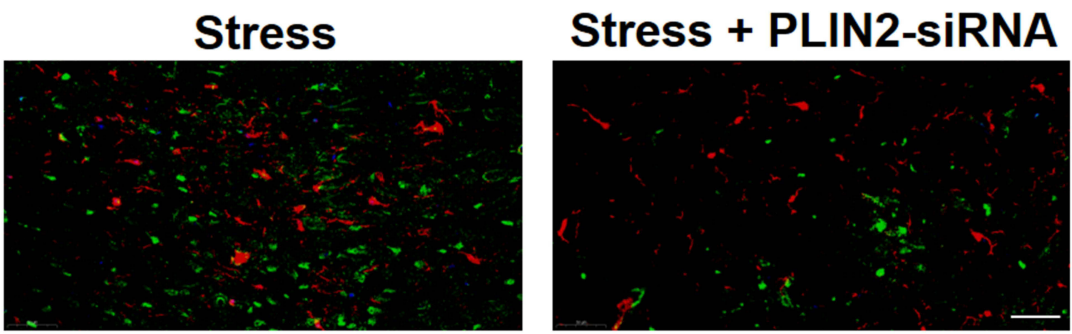

F

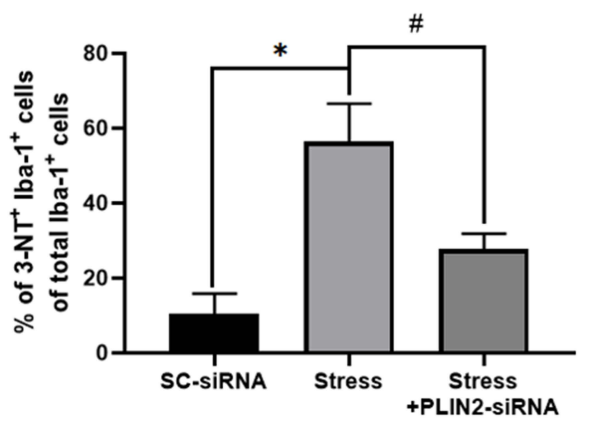

Figure 6 PLIN2 knock down alleviates RVLM oxidative/nitrosative stress in stressed rats. The representative double immunofluorescent staining shows the colocalization image of 4-HNE (an oxidative/nitrosative indicator) with lba-I (A), the quantification of 4-HNE immunopositive intensity (B), and the percent of 4-HNE ${ }^{+} / \mathrm{Iba}^{-} \mathrm{I}^{+}$cells in the RVLM of rats (C). The representative double immunofluorescent staining shows the colocalization image of 3-NT (a nitrosative indicator) with Iba-I (D), the quantification of 3-NT immunopositive intensity $(\mathbf{E})$, and the percent of $3-\mathrm{NT}^{+} / \mathrm{lba}^{-I^{+}}$cells in the RVLM of rats $(\mathbf{F})$. Scale bar $=50 \mu \mathrm{m}$. Data are presented as the mean $\pm \mathrm{SD}$. $\mathrm{n}=6-8,{ }^{*} \mathrm{p}<$ $0.05,{ }^{\#} \mathrm{p}<0.05$, t-test.

proportion in RVLM of stressed rats (Figure 8A and B). Furthermore, PLIN2 knockdown in RVLM reduced the serum norepinephrine (NE) levels, which reflected that peripheral sympathetic activity was reduced in stressed rats (Figure $8 \mathrm{C}$ ). Heart rate variability analysis showed that the LF/HF ratio decreased in stressed rats while central PLIN2 knockdown suppressed this decline
(Figure 8D). BP measurements showed that there was a significant rise in mean arterial blood pressure (MAP) of stressed rats, while PLIN2-siRNA attenuated the rise in MAP of stressed rats (Figure 8E). These data indicate that PLIN2 knockdown in RVLM ameliorates the cardiovascular sympathetic overactivation and high blood pressure in stressed rats. 
A

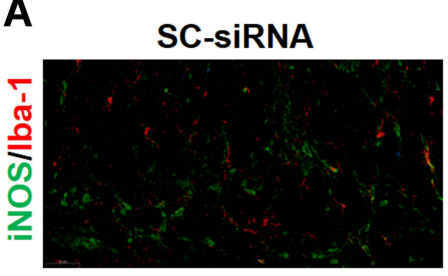

C

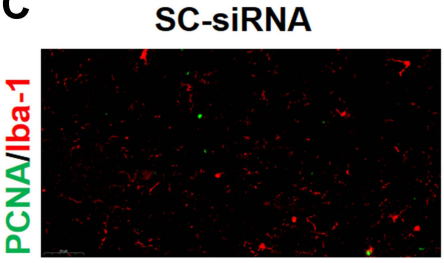

E

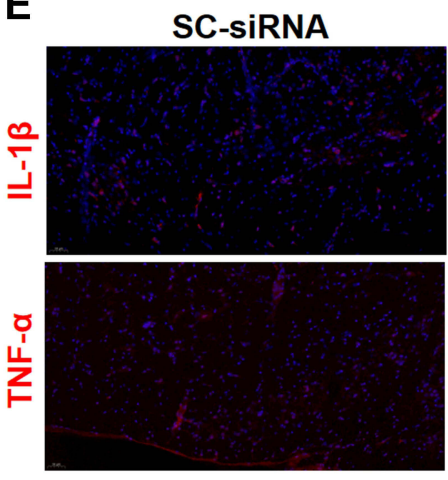

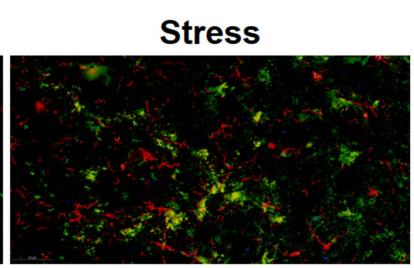

Stress

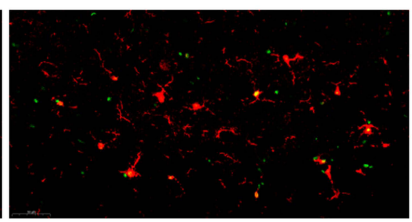

Stress

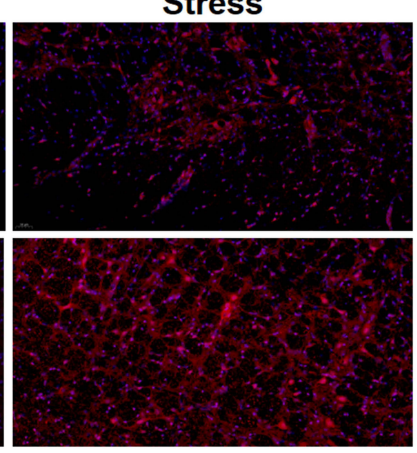

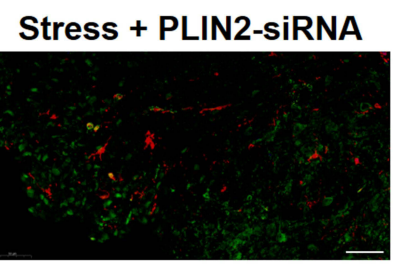

B

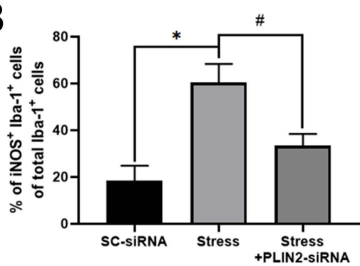

Stress + PLIN2-siRNA
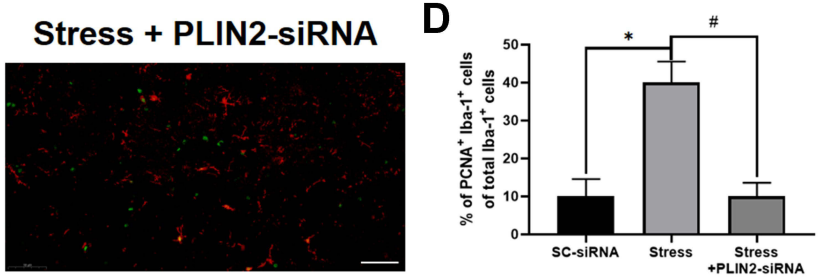

Stress + PLIN2-siRNA

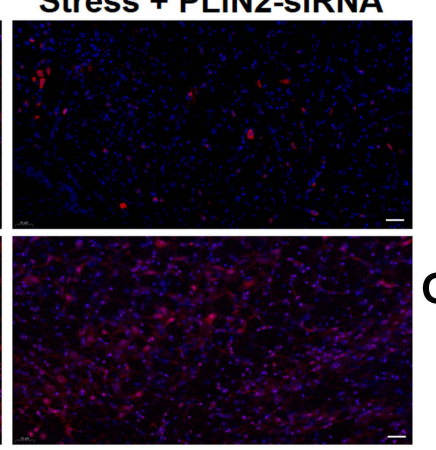

F

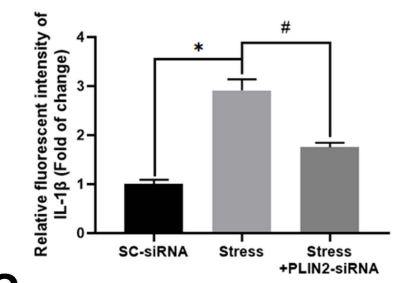

G

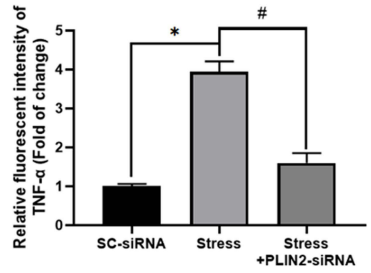

Figure 7 PLIN2 knock down attenuates RVLM microglial MI polarization and proliferation in stressed rats. The representative double immunofluorescent image of iNOS (MI polarization marker) with lba-I (A) co-localization and the percent of iNOS ${ }^{+} / \mathrm{lba}^{-I^{+}}$cells $(\mathbf{B})$ in the RVLM of rats. Scale bar $=50 \mu \mathrm{m}$. The representative double immunofluorescent image of colocalization of PCNA (proliferation marker) with lba-I (C) and the percent of PCNA $/ / \mathrm{lba}^{+} \mathrm{I}^{+}$cells $(\mathbf{D})$ in the RVLM of rats. Scale bar $=50 \mu \mathrm{m}$. The representative immunofluorescent image of IL-I $\beta$ (upper panel)/ TNF- $\alpha$ (lower panel) (E) and quantification of immunopositive intensity of IL-I $\beta$ (F)/ TNF- $\alpha$ (G) in the RVLM. Data are presented as the mean \pm SD. Scale bar $=50 \mu \mathrm{m} . \mathrm{n}=6-8,{ }^{*} \mathrm{p}<0.05,{ }^{\#} \mathrm{p}<0.05$, $t$-test.

\section{PLIN2 Knockdown Ameliorates Myocardial Injury in Stressed Rats}

Myocardial remodeling including cardiomyocyte hypertrophy and interstitial fibrosis is a process that develops in response to pathological stimuli such as pressure overload and neuroendocrine activation. To investigate myocardial remodeling in SIH rats, HE, Masson, and WGA staining were used, respectively, to evaluate the morphological changes of left ventricle in rats (Figure 9A). The increased myocardial fibrosis area (Figure 9B) and myocyte crosssectional area (Figure 9C) were bigger in stressed rats compared to that of sham group, while central PLIN2 knocked-down ameliorated myocardial fibrosis and hypertrophy in stress rats. Further, stressed rats showed increased heart weight (HW), heart weight /tibia length ratio $(\mathrm{HW} / \mathrm{TL})$, left ventricular weight $(\mathrm{LW})$, and left ventricular weight/tibia length ratio (LW/TL), while these increases were attenuated by microinjection of PLIN2siRNA into the RVLM (Figure 10A-D). Corresponding with the pathohistological alteration, we measured several key cardiac parameters of rats by echocardiography. Representative images of echocardiographic M-mode imaging are shown in Figure 10E. There was no significant change of heart rates (HRs), left ventricular ejection fraction (LVEF) and shortener fraction (LVFS) (Figure 10F$H$ ), Stressed rats exhibited a significant increase in the thickness of the end-diastolic posterior wall (LVPWd) compared with the sham rats, while PLIN2 knockdown 

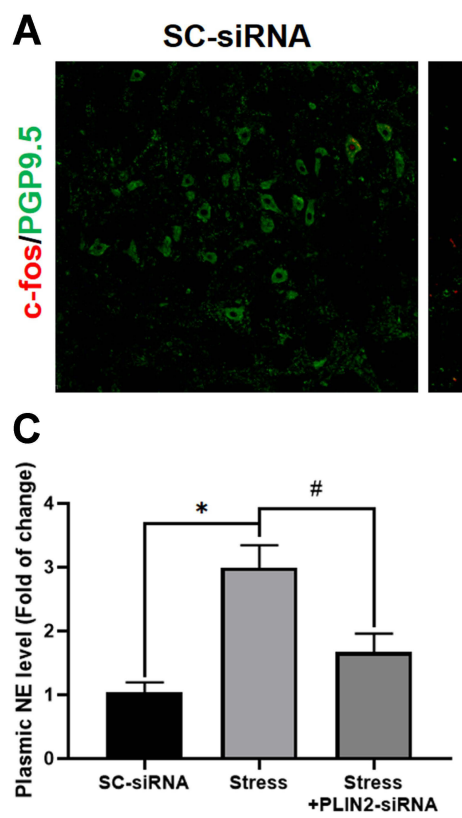

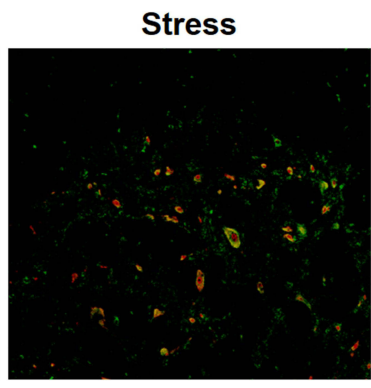

D

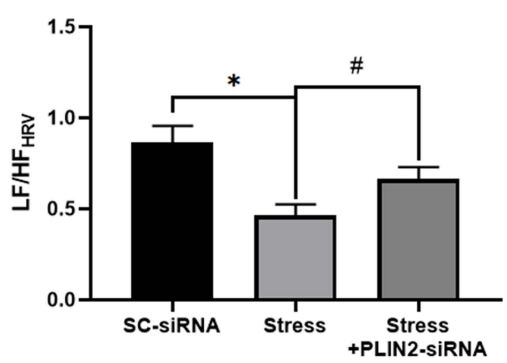

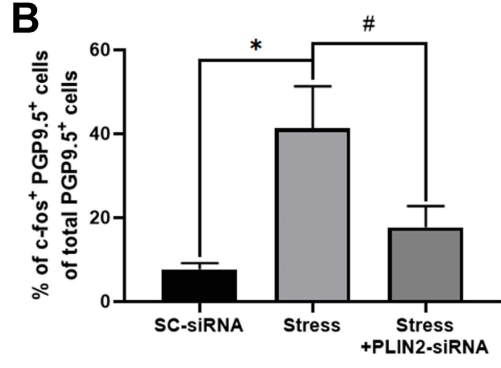

E

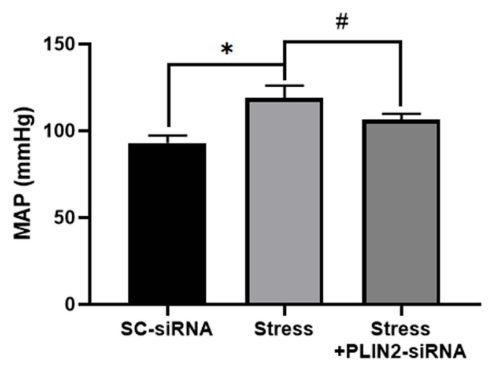

Figure 8 PLIN2 knock down alleviates sympathetic overactivation and lowers BP in stressed rats. The representative double immunofluorescent image of colocalization of c-fos (neuronal activation marker) with PGP9.5 (A) and the percent c-fos ${ }^{+} /$PGP9.5 $5^{+}$cells (B) in the RVLM. Scale bar $=100 \mu \mathrm{m}$. (C) The ELISA assay analysis shows that PLIN2 knock down in RVLM attenuates stress-induced plasmic NE increasing in rats. (D) The results of heart rate variability (HRV) analysis showed PLIN2 knock down in RVLM ameliorates stress-induced LF/HF increasing ratio of rats, which implies cardiac sympathetic/parasympathetic tones imbalance with the sympathetic overactivation. (E) Recorded and analyzed mean arterial blood pressure $(\mathrm{mmHg})$, data suggested that PLIN2-siRNA lower the stress-evoked hypertension in rats. Data are presented as the mean \pm SD. $\mathrm{n}=6-8,{ }^{*} \mathrm{p}<0.05,{ }^{\#} \mathrm{p}<0.05, t$-test.

in RVLM inhibit the above change (Figure 10I). All these laboratory evidence indicate that PLIN2 knockdown inhibit stress-induced myocardial injury.

\section{Discussion}

Microglial phenotypes have highlighted robust changes in the lipid and lipoprotein metabolism to meet its function, which profiles encountering the chronic stress remains unclear. In our present study, firstly, we found significant M1 polarization and microglia proliferation in the RVLM of stress rats, accompanied by increased LDs accumulation. Secondly, non-targeted lipid metabolomics results showed that the content of phosphatidylethanolamine (PE) in the RVLM region of stressed rats was significantly decreased. Thirdly, we observed activation of PLIN2, one of LDs proteins, led to accumulation of LDs, which caused the deficiency of PE. Fourthly, exogenous PE supplementation alleviates prorenin stress-induced oxidative/nitrosative stress in microglia. Finally, we found that central knockdown of PLIN2 alleviated stress-induced RVLM neuroinflammation, high BP and myocardial injury. Our research implied that the stress included LDs accumulation was mediated by PLIN2, thereby disturbed PE metabolism in microglia and its proliferation.
It was noted that $\mathrm{PE}$ is one of the most abundant phospholipid in eukaryotes, which participate in the regulation of phospholipid bilayer genesis, fusion, as well as fluidity, and is closely related to redox homeostasis, mitochondrial function, and endoplasmic reticulum stress (ERS). ${ }^{59}$ The decrease of PE has been reported in a variety of stress and metabolic strike models, which are closely related to inflammation, oxidative stress, mitochondrial dysfunction, and autophagy. ${ }^{60-62}$ In addition to PE another phospholipid phosphatidylcholine (PC), with the largest content, was also changed in the stressed rats. We found stress might lead to an increase in the ratio of PC/PE. Previous literature has reported that obesity and a variety of stresses lead to an increase in the ratio of $\mathrm{PC} /$ $\mathrm{PE}$, which is accompanied with the initiation of inflammation and the metabolic remodeling. ${ }^{63-66}$ It is important to note that $\mathrm{CCT} \alpha$, a rate-limiting enzyme for phospholipid synthesis, is not expressed in rat brain, and CCT $\beta$ expression is only detected in Purkinje cells. ${ }^{67}$ This suggests that the de novo synthesis of phospholipids is not the main pathway for most brain cells. Another one not to be overlooked is the conversion of $\mathrm{PE} / \mathrm{PC}$ via Phosphatidylethanolamine N-methyltransferase (PEMT), which causes declined PE and increased PC. ${ }^{68}$ It was known that catalytic activity of PEMT are involved in 
A
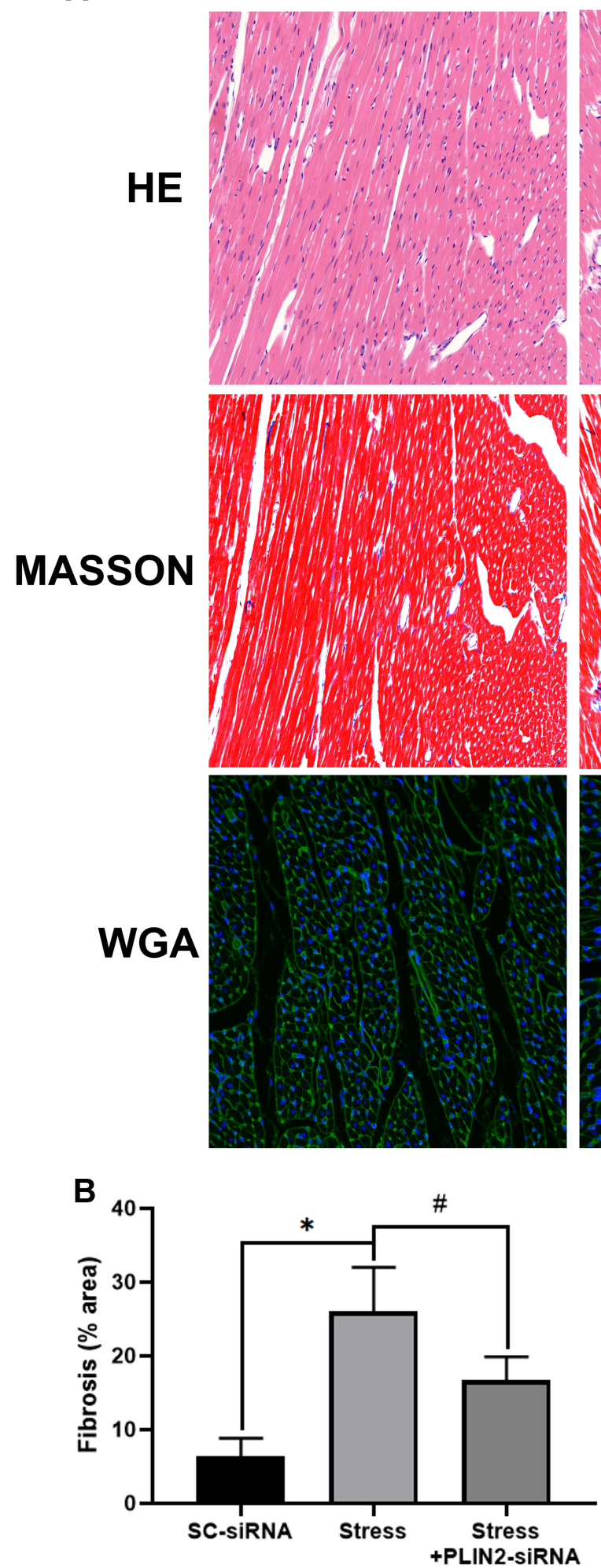

Stress Stress+PLIN2-siRNA
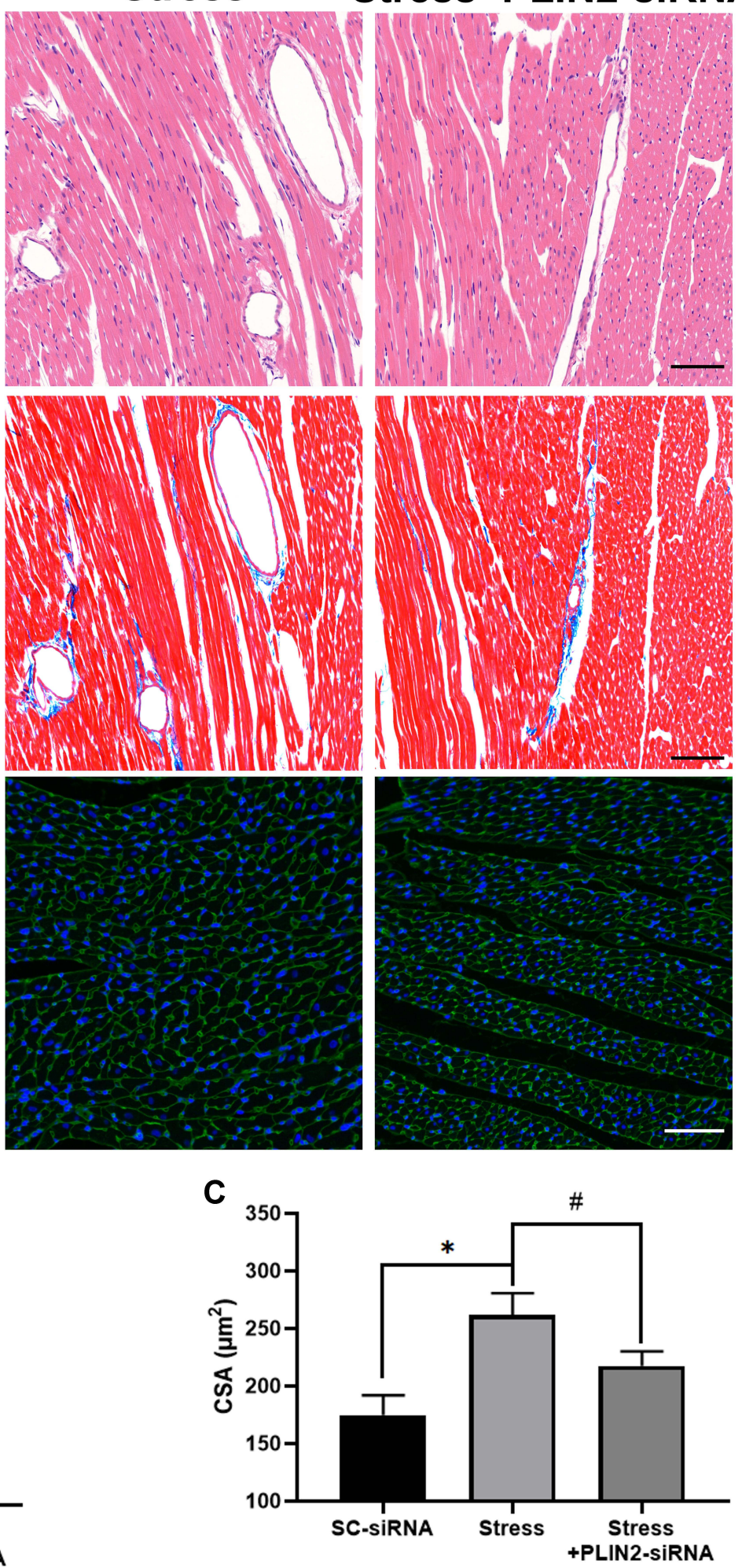

Figure 9 PLIN2 knock down ameliorates myocardial pathohistological alteration in stressed rats. The representative image of hematoxylin-eosin (HE) staining (the upper panel), Masson-trichrome staining (the middle panel) and WGA staining (the lower panel) of left ventricular tissue of heart. $(\mathbf{A})$ Scale bar $=50 \mu \mathrm{m}$. The quantification analysis of fibrosis area (B) and cross section area (C) show PLIN-siRNA attenuates myocardial fibrosis and hypertrophy in stressed rats. Data are presented as the mean \pm SD. $\mathrm{n}=6-8,{ }^{*} \mathrm{p}<0.05,{ }_{\mathrm{p}}^{\#}<0.05$, t-test. 
A

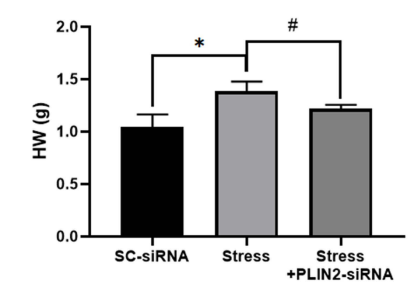

E

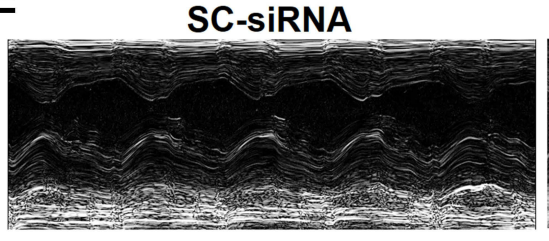

$\mathbf{F}$

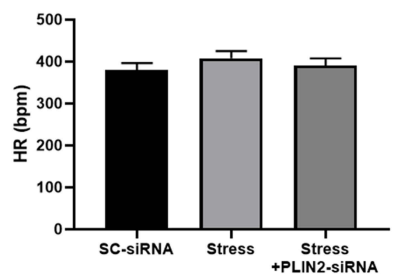

B

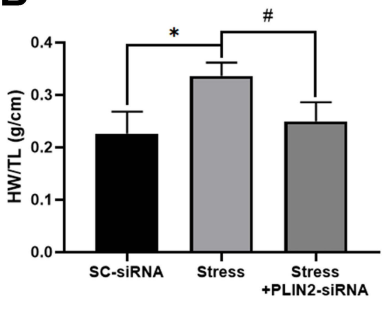

C

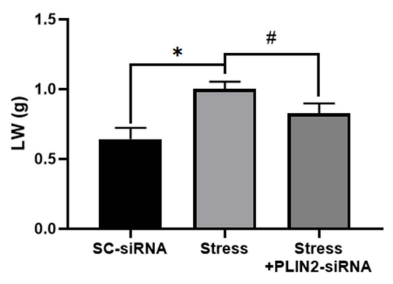

D

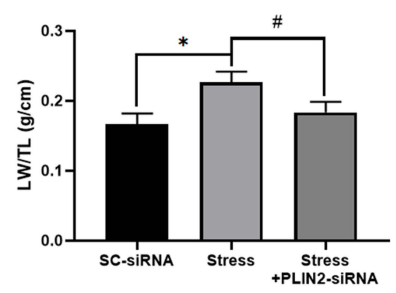

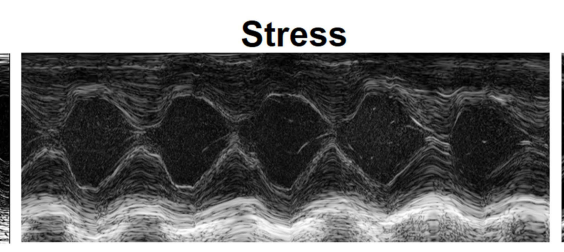

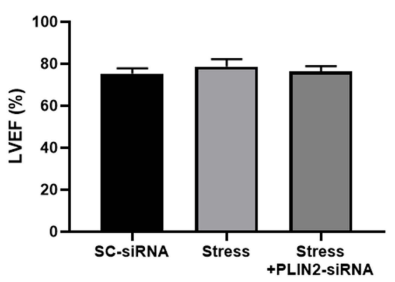

H

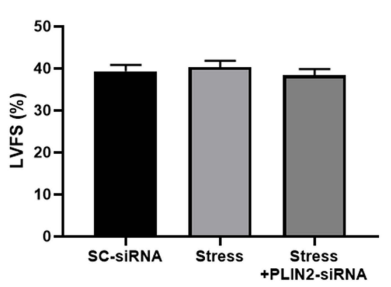

Stress+PLIN2-siRNA

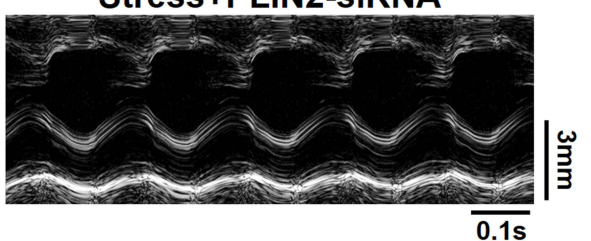

I

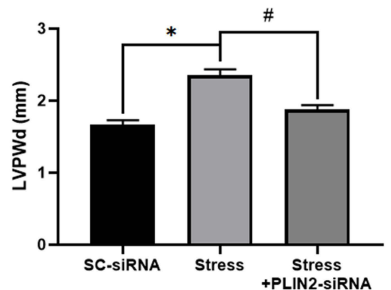

Figure 10 PLIN2 knock down ameliorates cardiac dysfunction in stressed rats. (A-D) The heart weight (HW), heart weight /tibia length ratio (HW/TL), left ventricular weight (LVW), and left ventricular weight /tibia length ratio (LVW/TL) are measured in rats of different group. The representative M-mode images of echocardiography is shown (E) and heart rates (HRs), left ventricular ejection fraction (LVEF), shorten fraction (LVFS) and the thickness of the end-diastolic posterior wall (LVPWd) are quantified $(\mathbf{F}-\mathbf{I})$. Data are presented as the mean \pm SD. $n=6-8,{ }^{*} p<0.05,{ }^{*} p<0.05, t$-test.

the development of Alzheimer's disease, obesity and nonalcoholic fatty liver disease. ${ }^{38,69,70}$ The PEMT-knockout mice on the high-fat diet appeared to have a greater increase in systolic blood pressure. ${ }^{71}$ All these suggest there are potential relationship between PE deficiency and autonomic nervous disorders.

Our study suggested that knocking down PLIN2 could partially restore PE content via reducing LDs in microglia stimulated by prorenin. As the lipid-storage organelle, LDs are also the integration platform of the lipid metabolism network. In particular, the perilipin protein represented by PLIN2 is involved in the degradation of LDs and utilization of neutral fatty acids, and the regulation of the interaction between LDs and other organelles. ${ }^{72-74}$ Our present study showed that in activated microglia, increased PLIN2 mediated LDs accumulation, while PLIN2 knockdown inhibited LDs accumulation, which is consistent with other investigations. In addition, previous studies also reported that the changes in PEMT activity are closely related to the fusion of LDs. Intervention of PE degradation mediated by PEMT and increase of PC synthesis can facilitate the fusion of LDs, suggesting a potential connection between degradation of LDs and increase of PE synthesis. ${ }^{75}$ Besides, what should not be ignored is that studies have reported that LDs act as the attachment interface of enzymes in phospho-lipid anabolism and regulate phospholipid production. ${ }^{76-78}$ Thus, follow-up studies need to further explore the molecular mechanism of LDs accumulation in microglia resulting in dysregulation of phospholipid biosynthesis and metabolism more precisely.

In our present study, we demonstrated that supplementation with exogenous PE blocked the inflammatory activation of microglia. Previous studies have reported that increased mitochondrial PE can stimulate function of respiratory chain proteins (including cytochrome c oxidase and succinate reductase), release mitochondrial aerobic respiration potential, increase ATP synthesis and improve cell metabolism. ${ }^{79}$ The lack of PE leads to mitochondrial fragmentation and excessive fusion, accompanied by mitochondrial structure disorder observed under transmission electron microscope. ${ }^{80} \mathrm{PE}$ deficiency impairs formation and/or membrane integration of respiratory super-complexes, which could be mitigated by $\mathrm{PE}$ supplementation. ${ }^{81}$ In addition, the role of $\mathrm{PE}$ in 
autophagy was noticed. PE-modified LC3 mediates the formation and transport of autophagosomes, which plays a crucial role in the initiation of selective autophagy and autophagosomes-lysosomes fusion. ${ }^{82} \mathrm{PE}$ deficiency directly leads to autophagy disruption, and the disorder of selective autophagy is closely related to the occurrence and development of mitochondrial and endoplasmic reticulum stress. Our previous publication shows that oxidative stress is an essential event for triggering mitochondrial dysfunction and autophagy blockage in the stressed microglia. The present study further confirmed that PE supplementation alleviated mitoROS production and accumulation of oxidative/nitrosative stress products in prorenin-stimulated microglia.

Oxidative stress/nitrosative stress in the sympathetic center is the key mechanism of sympathetic overactivation in hypertension and other cardiovascular events, including myocardial infarction. The RAS (renin-angiotensin system) is the most important trigger factor of oxidative/ nitrosative stress in the cardiovascular center. ${ }^{13,83}$ Microglia are the main cells that produce ROS/NO in the brain under pathological stimulation. ${ }^{84,85}$ Studies have confirmed that various kinds of pro-hypertensive stress induce the activation of local RAS system in the sympathetic center to induce neuroinflammation and oxidative stress, and the circulating RAS components also stimulate the sympathetic activity through Subfornical organparaventricular nucleus-RVLM or directly activate the sympathetic center through blood-brain barrier microleakage. ${ }^{86,87}$ Alleviating RAS effect in brain regions such as RVLM can significantly inhibit the increase of sympathetic output and BP caused by various kinds of stress. However, the molecular mechanism of oxidative/nitrosative stress triggered by the RAS system has not been fully explored. AT1 receptor activation-induced NADPH oxidases (NOXs) activation and iNOS expression are common mechanisms observed in various hypertensive models and myocardial infarction stress models. ${ }^{88,89}$ Attenuating the increased production of mitoROS due to mitochondrial dysfunction is another promising strategy for blocking downstream AT1 molecular events.

Our previous studies confirmed that prorenin, an important component of the RAS system, induces oxidative stress and NOXs activation through prorenin receptor, leading to M1 polarization of microglia in the RVLM, which is involved in the increase of sympathetic output in stress-induced hypertension rats. ${ }^{15}$ In our present study, we further found that PLIN2-mediated LDs increase led to oxidative/nitrosative stress in the RVLM of stressed rats, while PE supplementation blocked the prorenin-induced increase of ROS/nitrosative stress products. These results indicate that PLIN2-mediated PE deficiency in the sympathetic center is involved in the development of stressinduced hypertension. This study provided a new therapeutic strategy for interfering stress-induced hypertension and hypertensive heart injury.

\section{Conclusion}

Collectively, we demonstrated that inhibition of the PLIN2 in the RVLM alleviates oxidative/nitrosative stress, dampening sympathetic overdrive and protecting the heart from hypertrophy and fibrosis in stressed rats.

\section{Abbreviations}

3-NT, 3-nitrotyrosine; 4-HNE, 4-Hydroxynonenal; CSA, Cross-section area; CTP, phosphocholine cytidylyltransferase; ECG, Echocardiogram; GSH, Glutathione; H \& E staining, Hematoxylin and Eosin Staining; HPA, Hypothalamic-pituitary-adrenal axis; HR, Heart rate; HRV, Heart rate variability; HW, Heart weight; HW/TL, Heart weight/Tibia length ratio; IL-1 $\beta$, Interleukin-1 $\beta$; iNOS, inducible Nitric oxide synthase; LDs, Lipid droplets; LF/HF, Low Frequency/High Frequency; LVEF, left ventricular ejection fraction; LVFS, Left ventricular shortener fraction; LVPWd, the end-diastolic posterior wall of left ventricular; LW, left ventricular weight; LW/TL, left ventricular weight/tibia length ratio; MAP, Mean arterial blood pressure; MDA, Malondialdehyde; mitoROS, Mitochondrial Reactive Oxygen Species; NE, Norepinephrine; NLRP3, NLR family pyrin domain containing 3; NO, nitric oxide; PC, Phosphatidylcholine; PCNA, Proliferating Cell Nuclear Antigen; PE, Phosphatidylethanolamine; PEMT, Phosphatidylethanolamine N-methyltransferase; PICs, proinflammatory cytokines; PLIN2, Perilipin 2; PVN, Paraventricular nucleus; RAS, Renin-angiotensin system; RVLM, Rostral ventrolateral medulla; SEM, mean \pm standard error of the mean; SIH, Stress-induced hypertension; TLRs, Toll-like receptors.

\section{Acknowledgments}

This work was supported by the National Natural Science Foundation of China [81770423, 32071111, 31871151, 31571171] and Special project of Science and Technology Plan of Shaoxing Science and Technology Bureau (No. 2020B33004). 


\section{Disclosure}

The authors report no conflicts of interest in this work.

\section{References}

1. Munakata M. Clinical significance of stress-related increase in blood pressure: current evidence in office and out-of-office settings. Hypertens Res. 2018;41(8):553-569. doi:10.1038/s41440-018-0053-1

2. Liu MY, Li N, Li WA, Khan H. Association between psychosocial stress and hypertension: a systematic review and meta-analysis. Neurol Res. 2017;39(6):573-580. doi:10.1080/01616412.2017.1317904

3. Frank MG, Fonken LK, Watkins LR, Maier SF. Microglia: neuroimmune-sensors of stress. Semin Cell Dev Biol. 2019;94:176-185. doi:10.1016/j.semcdb.2019.01.001

4. Madore C, Yin Z, Leibowitz J, Butovsky O. Microglia, lifestyle stress, and neurodegeneration. Immunity. 2020;52(2):222-240. doi:10.1016/j.immuni.2019.12.003

5. Du D, Hu L, Wu J, et al. Neuroinflammation contributes to autophagy flux blockage in the neurons of rostral ventrolateral medulla in stress-induced hypertension rats. J Neuroinflammation. 2017;14 (1):169. doi:10.1186/s12974-017-0942-2

6. Dworak M, Stebbing M, Kompa AR, Rana I, Krum H, Badoer E. Attenuation of microglial and neuronal activation in the brain by ICV minocycline following myocardial infarction. Auton Neurosci. 2014;185:43-50. doi:10.1016/j.autneu.2014.03.007

7. Zubcevic J, Santisteban MM, Perez PD, et al. A single angiotensin II hypertensive stimulus is associated with prolonged neuronal and immune system activation in Wistar-Kyoto rats. Front Physiol. 2017;8:592. doi:10.3389/fphys.2017.00592

8. Kishi T, Hirooka Y, Konno S, Ogawa K, Sunagawa K. Angiotensin II type 1 receptor-activated caspase-3 through ras/mitogen-activated protein kinase/extracellular signal-regulated kinase in the rostral ventrolateral medulla is involved in sympathoexcitation in stroke-prone spontaneously hypertensive rats. Hypertension. 2010;55(2):291-297. doi:10.1161/HYPERTENSIONAHA.109.138636

9. Mohsin M, Souza LAC, Aliabadi S, et al. Increased (Pro)renin receptor expression in the hypertensive human brain. Front Physiol. 2020;11:606811. doi:10.3389/fphys.2020.606811

10. Wu KLH, Wu CW, Chao YM, Hung CY, Chan JYH. Impaired Nrf2 regulation of mitochondrial biogenesis in rostral ventrolateral medulla on hypertension induced by systemic inflammation. Free Radic Biol Med. 2016;97:58-74. doi:10.1016/j.freeradbiomed.2016.05.012

11. Jiang L, Zhou X, Yang H, et al. Upregulation of AT1 receptor mediates a pressor effect through ROS-SAPK/JNK signaling in glutamatergic neurons of rostral ventrolateral medulla in rats with stress-induced hypertension. Front Physiol. 2019;9:1860. doi:10.3389/fphys.2018.01860

12. Chan JYH, Chan SHH. Differential impacts of brain stem oxidative stress and nitrosative stress on sympathetic vasomotor tone. Pharmacol Ther. 2019;201:120-136. doi:10.1016/j.pharmthera.2019.05.015

13. Hu L, Zhang S, Ooi K, et al. Microglia-derived NLRP3 activation mediates the pressor effect of prorenin in the rostral ventrolateral medulla of stress-induced hypertensive rats. Neurosci Bull. 2020;36 (5):475-492. doi:10.1007/s12264-020-00484-9

14. Zhang S, Hu L, Jiang J, et al. HMGB1/RAGE axis mediates stress-induced RVLM neuroinflammation in mice via impairing mitophagy flux in microglia. $J$ Neuroinflammation. 2020;17(1):15. doi:10.1186/s12974-019-1673-3

15. Hu L, Zhang S, Wen H, et al. Melatonin decreases M1 polarization via attenuating mitochondrial oxidative damage depending on UCP2 pathway in prorenin-treated microglia. PLoS One. 2019;14(2): e0212138. doi:10.1371/journal.pone.0212138

16. Li Y, Wei B, Liu X, Shen XZ, Shi P. Microglia, autonomic nervous system, immunity and hypertension: Is there a link? Pharmacol Res. 2020;155:104451. doi:10.1016/j.phrs.2019.104451
17. Shen $\mathrm{XZ}, \mathrm{Li} \mathrm{Y}, \mathrm{Li} \mathrm{L}$, et al. Microglia participate in neurogenic regulation of hypertension. Hypertension. 2015;66(2):309-316. doi:10.1161/HYPERTENSIONAHA. 115.05333

18. Olmos-Alonso A, Schetters ST, Sri S, et al. Pharmacological targeting of CSF1R inhibits microglial proliferation and prevents the progression of Alzheimer's-like pathology. Brain. 2016;139(Pt 3):891-907. doi:10.1093/brain/awv379

19. Feng L, Murugan M, Bosco DB, et al. Microglial proliferation and monocyte infiltration contribute to microgliosis following status epilepticus. Glia. 2019;67(8):1434-1448. doi:10.1002/glia.23616

20. Ziko I, De Luca S, Dinan T, et al. Neonatal overfeeding alters hypothalamic microglial profiles and central responses to immune challenge long-term. Brain Behav Immun. 2014;41:32-43. doi:10.1016/j.bbi.2014.06.014

21. Schubert I, Ahlbrand R, Winter A, Vollmer L, Lewkowich I, Sah R. Enhanced fear and altered neuronal activation in forebrain limbic regions of CX3CR1-deficient mice. Brain Behav Immun. 2018;68:34-43. doi:10.1016/j.bbi.2017.09.013

22. Biancardi VC, Stranahan AM, Krause EG, de Kloet AD, Stern JE. Cross talk between AT1 receptors and toll-like receptor 4 in microglia contributes to angiotensin II-derived ROS production in the hypothalamic paraventricular nucleus. Am J Physiol Heart Circ Physiol. 2016;310(3):H404-H415. doi:10.1152/ ajpheart.00247.2015

23. Xu ML, Yu XJ, Zhao JQ, et al. Calcitriol ameliorated autonomic dysfunction and hypertension by down-regulating inflammation and oxidative stress in the paraventricular nucleus of SHR. Toxicol Appl Pharmacol. 2020;394:114950. doi:10.1016/j.taap.2020.114950

24. Marschallinger $J$, Iram $T$, Zardeneta $M$, et al. Lipid-dropletaccumulating microglia represent a dysfunctional and proinflammatory state in the aging brain. Nat Neurosci. 2020;23(2):194-208. doi:10.1038/s41593-019-0566-1

25. Kim ST, Kyung EJ, Suh JS, et al. Phosphatidylcholine attenuated docetaxel-induced peripheral neurotoxicity in rats. Drug Chem Toxicol. 2018;41(4):476-485. doi:10.1080/01480545.2017.1390580

26. Karunakaran I, van Echten-deckert G. Sphingosine 1-phosphate A double edged sword in the brain. Biochim Biophys Acta Biomembr. 2017;1859(9 Pt $\quad$ B):1573-1582. doi:10.1016/j. bbamem.2017.03.008

27. Rong X, Albert CJ, Hong C, et al. LXRs regulate ER stress and inflammation through dynamic modulation of membrane phospholipid composition. Cell Metab. 2013;18(5):685-697. doi:10.1016/j. cmet.2013.10.002

28. Snider SA, Margison KD, Ghorbani P, et al. Choline transport links macrophage phospholipid metabolism and inflammation. $J$ Biol Chem. 2018;293(29):11600-11611. doi:10.1074/jbc.RA118.003180

29. Karagiannis F, Masouleh SK, Wunderling K, et al. Lipid-droplet formation drives Pathogenic group 2 innate lymphoid cells in airway inflammation. Immunity. 2020;52(4):620-634.e6. doi:10.1016/j. immuni.2020.03.003

30. Yang B, Li R, Michael Greenlief C, et al. Unveiling anti-oxidative and anti-inflammatory effects of docosahexaenoic acid and its lipid peroxidation product on lipopolysaccharide-stimulated BV-2 microglial cells. J Neuroinflammation. 2018;15(1):202. doi:10.1186/ s12974-018-1232-3

31. Wenzel TJ, Ranger AL, McRae SA, Klegeris A. Extracellular cardiolipin modulates microglial phagocytosis and cytokine secretion in a toll-like receptor (TLR) 4-dependent manner. J Neuroimmunol. 2021;353:577496. doi:10.1016/j.jneuroim.2021.577496

32. Oliveira TG, Chan RB, Bravo FV, et al. The impact of chronic stress on the rat brain lipidome. Mol Psychiatry. 2016;21(1):80-88. doi: $10.1038 / \mathrm{mp} .2015 .14$

33. Faria R, Santana MM, Aveleira CA, et al. Alterations in phospholipidomic profile in the brain of mouse model of depression induced by chronic unpredictable stress. Neuroscience. 2014;273:1-11. doi:10.1016/j.neuroscience.2014.04.042 
34. Lee S, Norheim F, Gulseth HL, et al. Skeletal muscle phosphatidylcholine and phosphatidylethanolamine respond to exercise and influence insulin sensitivity in men. Sci Rep. 2018;8(1):6531. doi:10.1038/ s41598-018-24976- $\mathrm{x}$

35. Senoo N, Miyoshi N, Goto-Inoue N, et al. PGC-1 $\alpha$-mediated changes in phospholipid profiles of exercise-trained skeletal muscle. J Lipid Res. 2015;56(12):2286-2296. doi:10.1194/jlr.M060533

36. van der Veen JN, Kennelly JP, Wan S, Vance JE, Vance DE, Jacobs RL. The critical role of phosphatidylcholine and phosphatidylethanolamine metabolism in health and disease. Biochim Biophys Acta Biomembr. 2017;1859(9 Pt B):1558-1572. doi:10.1016/j. bbamem.2017.04.006

37. Arendt BM, Ma DW, Simons B, et al. Nonalcoholic fatty liver disease is associated with lower hepatic and erythrocyte ratios of phosphatidylcholine to phosphatidylethanolamine. Appl Physiol Nutr Metab. 2013;38(3):334-340. doi:10.1139/apnm-2012-0261

38. Wan S, van der Veen JN, Bakala N'Goma JC, Nelson RC, Vance DE, Jacobs RL. Hepatic PEMT activity mediates liver health, weight gain, and insulin resistance. FASEB J. 2019;33(10):10986-10995. doi:10.1096/fj.201900679R

39. Donoso F, Schverer M, Rea K, et al. Neurobiological effects of phospholipids in vitro: relevance to stress-related disorders. Neurobiol Stress. 2020;13:100252. doi:10.1016/j.ynstr.2020.100252

40. Sanchez-Lopez E, Zhong Z, Stubelius A, et al. Choline uptake and metabolism modulate macrophage IL-1 $\beta$ and IL-18 production. Cell Metab. 2019;29(6):1350-1362.e7. doi:10.1016/j.cmet.2019.03.011

41. Di Gioia M, Spreafico R, Springstead JR, et al. Endogenous oxidized phospholipids reprogram cellular metabolism and boost hyperinflammation. Nat Immunol. 2020;21(1):42-53. doi:10.1038/ s41590-019-0539-2

42. Olzmann JA, Carvalho P. Dynamics and functions of lipid droplets. Nat Rev Mol Cell Biol. 2019;20(3):137-155. doi:10.1038/s41580018-0085-z

43. Kimmel AR, Sztalryd C. The perilipins: major cytosolic lipid droplet-associated proteins and their roles in cellular lipid storage, mobilization, and systemic homeostasis. Annu Rev Nutr. 2016;36 (1):471-509. doi:10.1146/annurev-nutr-071813-105410

44. Qiu B, Ackerman D, Sanchez DJ, et al. HIF2 $\alpha$-dependent lipid storage promotes endoplasmic reticulum homeostasis in clear-cell renal cell carcinoma. Cancer Discov. 2015;5(6):652-667. doi:10.1158/2159-8290.CD-14-1507

45. Zhang S, Liu G, Xu C, et al. Perilipin 1 mediates lipid metabolism homeostasis and inhibits inflammatory cytokine synthesis in bovine adipocytes. Front Immunol. 2018;9:467. doi:10.3389/ fimmu.2018.00467

46. Tan Y, Jin Y, Wu X, Ren Z. PSMD1 and PSMD2 regulate HepG2 cell proliferation and apoptosis via modulating cellular lipid droplet metabolism. BMC Mol Biol. 2019;20(1):24. doi:10.1186/s12867019-0141-z

47. Polycarpou-Schwarz M, Groß M, Mestdagh P, et al. The cancer-associated microprotein CASIMO1 controls cell proliferation and interacts with squalene epoxidase modulating lipid droplet formation. Oncogene. 2018;37(34):4750-4768. doi:10.1038/s41388018-0281-5

48. Li LL, Wang D, Ge CY, Yu L, Zhao JL, Ma HT. Dehydroepiandrosterone reduced lipid droplet accumulation via inhibiting cell proliferation and improving mitochondrial function in primary chicken hepatocytes. Physiol Res. 2018;67(3):443-456. doi: $10.33549 /$ physiolres.933769

49. Tsai TH, Chen E, Li L, et al. The constitutive lipid droplet protein PLIN2 regulates autophagy in liver. Autophagy. 2017;13 (7):1130-1144. doi:10.1080/15548627.2017.1319544

50. Kaushik S, Cuervo AM. AMPK-dependent phosphorylation of lipid droplet protein PLIN2 triggers its degradation by CMA. Autophagy. 2016;12(2):432-438. doi:10.1080/15548627.2015.1124226
51. Kaushik S, Cuervo AM. Degradation of lipid droplet-associated proteins by chaperone-mediated autophagy facilitates lipolysis. Nat Cell Biol. 2015;17(6):759-770. doi:10.1038/ncb3166

52. Imamura M, Inoguchi $\mathrm{T}$, Ikuyama $\mathrm{S}$, et al. ADRP stimulates lipid accumulation and lipid droplet formation in murine fibroblasts. Am J Physiol Endocrinol Metab. 2002;283(4):E775-E783.

53. Lalancette-Hébert M, Moquin A, Choi AO, Kriz J, Maysinger D. Lipopolysaccharide-QD micelles induce marked induction of TLR2 and lipid droplet accumulation in olfactory bulb microglia. Mol Pharm. 2010;7(4):1183-1194. doi:10.1021/mp1000372

54. Shimabukuro MK, Langhi LG, Cordeiro I, et al. Lipid-laden cells differentially distributed in the aging brain are functionally active and correspond to distinct phenotypes. Sci Rep. 2016;6(1):23795. doi:10.1038/srep23795

55. Paxinos G, Watson CR, Emson PC. AChE-stained horizontal sections of the rat brain in stereotaxic coordinates. J Neurosci Methods. 1980;3(2):129-149. doi:10.1016/0165-0270(80)90021-7

56. Wu J, Zhang Y, Wu Q, et al. Integrative analyses of myocardial lipidome and proteome implicate mitochondrial dysfunction in lethal ventricular tachyarrhythmia (LVTA) induced by acute myocardial ischemia (AMI). J Proteomics. 2019;197:14-22. doi:10.1016/j. jprot.2019.01.021

57. Chen QQ, Wang FX, Cai YY, et al. Untargeted metabolomics and lipidomics uncovering the cardioprotective effects of Huanglian Jiedu decoction on pathological cardiac hypertrophy and remodeling. J Ethnopharmacol. 2021;270:113646. doi:10.1016/j.jep.2020.113646

58. Schiattarella GG, Altamirano F, Tong D, et al. Nitrosative stress drives heart failure with preserved ejection fraction. Nature. 2019;568(7752):351-356. doi:10.1038/s41586-019-1100-z

59. Calzada E, Onguka O, Claypool SM. Phosphatidylethanolamine metabolism in health and disease. Int Rev Cell Mol Biol. 2016;321:29-88. doi:10.1016/bs.ircmb.2015.10.001

60. Patel D, Witt SN. Ethanolamine and phosphatidylethanolamine: partners in health and disease. Oxid Med Cell Longev. 2017;2017:4829180. doi:10.1155/2017/4829180

61. Guo L, Chen Z, Cox BE, et al. Phosphatidylethanolamines modified by $\gamma$-ketoaldehyde $(\gamma \mathrm{KA})$ induce endoplasmic reticulum stress and endothelial activation. J Biol Chem. 2011;286(20):18170-18180. doi:10.1074/jbc.M110.213470

62. Trentzsch M, Nyamugenda E, Miles TK, et al. Delivery of phosphatidylethanolamine blunts stress in hepatoma cells exposed to elevated palmitate by targeting the endoplasmic reticulum. Cell Death Discov. 2020;6(1):8. doi:10.1038/s41420-020-0241-z

63. van der Veen JN, Lingrell S, da Silva RP, Jacobs RL, Vance DE. The concentration of phosphatidylethanolamine in mitochondria can modulate ATP production and glucose metabolism in mice. Diabetes. 2014;63(8):2620-2630. doi:10.2337/db13-0993

64. Hang J, Liang S, Ning R, et al. PM2.5-induced inflammation and lipidome alteration associated with the development of atherosclerosis based on a targeted lipidomic analysis. Environ Int. 2020;136:105444. doi:10.1016/j.envint.2019.105444

65. Wiswedel I, Gardemann A, Storch A, Peter D, Schild L. Degradation of phospholipids by oxidative stress-exceptional significance of cardiolipin. Free Radic Res. 2010;44(2):135-145. doi:10.3109/ 10715760903352841

66. Ye C, Sutter BM, Wang Y, Kuang Z, Tu BP. A metabolic function for phospholipid and histone methylation. Mol Cell. 2017;66(2):180193.e8. doi:10.1016/j.molcel.2017.02.026

67. Pati S, Ingram LM, Sun MK, Wagner JJ, Cummings BS. Localization and expression of CTP: phosphocholine cytidylyltransferase in rat brain following cocaine exposure. J Chem Neuroanat. 2019;96:1-6. doi:10.1016/j.jchemneu.2018.10.006

68. Morita SY, Takeuchi A, Kitagawa S. Functional analysis of two isoforms of phosphatidylethanolamine N-methyltransferase. Biochem J. 2010;432(2):387-398. doi:10.1042/BJ20100490 
69. Guan ZZ, Wang YN, Xiao KQ, Hu PS, Liu JL. Activity of phosphatidylethanolamine-N-methyltransferase in brain affected by Alzheimer's disease. Neurochem Int. 1999;34(1):41-47. doi:10.1016/S0197-0186(98)00068-0

70. Watanabe M, Nakatsuka A, Murakami K, et al. Pemt deficiency ameliorates endoplasmic reticulum stress in diabetic nephropathy. PLoS One. 2014;9(3):e92647. doi:10.1371/journal.pone.0092647

71. Gao X, van der Veen JN, Fernandez-Patron C, Vance JE, Vance DE, Jacobs RL. Insufficient glucose supply is linked to hypothermia upon cold exposure in high-fat diet-fed mice lacking PEMT. J Lipid Res. 2015;56(9):1701-1710. doi:10.1194/jlr.M059287

72. Takahashi Y, Shinoda A, Kamada H, Shimizu M, Inoue J, Sato R. Perilipin2 plays a positive role in adipocytes during lipolysis by escaping proteasomal degradation. Sci Rep. 2016;6(1):20975. doi:10.1038/srep20975

73. Makino A, Hullin-Matsuda F, Murate M, et al. Acute accumulation of free cholesterol induces the degradation of perilipin 2 and Rab18-dependent fusion of ER and lipid droplets in cultured human hepatocytes. Mol Biol Cell. 2016;27(21):3293-3304. doi:10.1091/ mbc.E15-10-0730

74. Schulze RJ, Krueger EW, Weller SG, et al. Direct lysosome-based autophagy of lipid droplets in hepatocytes. Proc Natl Acad Sci U S A. 2020;117(51):32443-32452. doi:10.1073/pnas.2011442117

75. Hörl G, Wagner A, Cole LK, et al. Sequential synthesis and methylation of phosphatidylethanolamine promote lipid droplet biosynthesis and stability in tissue culture and in vivo. J Biol Chem. 2011;286 (19):17338-17350. doi:10.1074/jbc.M111.234534

76. Aitchison AJ, Arsenault DJ, Ridgway ND. Nuclear-localized CTP: phosphocholine cytidylyltransferase $\alpha$ regulates phosphatidylcholine synthesis required for lipid droplet biogenesis. Mol Biol Cell. 2015;26(16):2927-2938. doi:10.1091/mbc.E15-03-0159

77. Sołtysik K, Ohsaki Y, Tatematsu T, Cheng J, Fujimoto T. Nuclear lipid droplets derive from a lipoprotein precursor and regulate phosphatidylcholine synthesis. Nat Commun. 2019;10(1):473. doi:10.1038/s41467-019-08411-X

78. Krahmer N, Guo Y, Wilfling F, et al. Phosphatidylcholine synthesis for lipid droplet expansion is mediated by localized activation of CTP: phosphocholinecytidylyltransferase. Cell Metab. 2011;14 (4):504-515. doi:10.1016/j.cmet.2011.07.013

79. Heden TD, Johnson JM, Ferrara PJ, et al. Mitochondrial PE potentiates respiratory enzymes to amplify skeletal muscle aerobic capacity. Sci Adv. 2019;5(9):eaax8352. doi:10.1126/sciadv.aax8352
80. Tasseva G, Bai HD, Davidescu M, Haromy A, Michelakis E, Vance JE. Phosphatidylethanolamine deficiency in Mammalian mitochondria impairs oxidative phosphorylation and alters mitochondrial morphology. J Biol Chem. 2013;288(6):4158-4173. doi:10.1074/jbc. M112.434183

81. Öttinger L, Horvath SE, Kleinschroth T, et al. Phosphatidylethanolamine and cardiolipin differentially affect the stability of mitochondrial respiratory chain supercomplexes. $\mathrm{J} \mathrm{Mol}$ Biol. 2012;423(5):677-686. doi:10.1016/j.jmb.2012.09.001

82. Rockenfeller P, Koska M, Pietrocola F, et al. Phosphatidylethanolamine positively regulates autophagy and longevity. Cell Death Differ. 2015;22 (3):499-508. doi:10.1038/cdd.2014.219

83. Campese VM, Shaohua Y, Huiquin Z. Oxidative stress mediates angiotensin II-dependent stimulation of sympathetic nerve activity. Hypertension. 2005;46(3):533-539. doi:10.1161/01.HYP.0000179088. 57586.26

84. Wang $\mathrm{Y}, \mathrm{Hu} \mathrm{H}$, Yin $\mathrm{J}$, et al. TLR4 participates in sympathetic hyperactivity Post-MI in the PVN by regulating NF- $\kappa \mathrm{B}$ pathway and ROS production. Redox Biol. 2019;24:101186. doi:10.1016/j. redox.2019.101186

85. Nicolas S, Cazareth J, Zarif H, et al. Globular adiponectin limits microglia pro-inflammatory phenotype through an AdipoR1/NF- $\kappa \mathrm{B}$ signaling pathway. Front Cell Neurosci. 2017;11:352. doi:10.3389/ fncel.2017.00352

86. Osborn JW, Fink GD, Sved AF, Toney GM, Raizada MK. Circulating angiotensin II and dietary salt: converging signals for neurogenic hypertension. Curr Hypertens Rep. 2007;9(3):228-235. doi:10.1007/ s11906-007-0041-3

87. Wang HW, Huang BS, White RA, Chen A, Ahmad M, Leenen FH. Mineralocorticoid and angiotensin II type 1 receptors in the subfornical organ mediate angiotensin II - induced hypothalamic reactive oxygen species and hypertension. Neuroscience. 2016;329:112-121. doi:10.1016/j.neuroscience.2016.04.050

88. Zhang Y, Yu XJ, Chen WS, et al. Exercise training attenuates renovascular hypertension partly via RAS- ROS- glutamate pathway in the hypothalamic paraventricular nucleus. Sci Rep. 2016;6(1):37467. doi:10.1038/srep37467

89. Huber MJ, Basu R, Cecchettini C, Cuadra AE, Chen QH, Shan Z. Activation of the (pro)renin receptor in the paraventricular nucleus increases sympathetic outflow in anesthetized rats. Am J Physiol Heart Circ Physiol. 2015;309(5):H880-H887. doi:10.1152/ajpheart.00095.2015
Journal of Inflammation Research

\section{Publish your work in this journal}

The Journal of Inflammation Research is an international, peerreviewed open-access journal that welcomes laboratory and clinical findings on the molecular basis, cell biology and pharmacology of inflammation including original research, reviews, symposium reports, hypothesis formation and commentaries on: acute/chronic inflammation; mediators of inflammation; cellular processes; molecular mechanisms; pharmacology and novel anti-inflammatory drugs; clinical conditions involving inflammation. The manuscript management system is completely online and includes a very quick and fair peerreview system. Visit http://www.dovepress.com/testimonials.php to read real quotes from published authors. 